\title{
La variabilidad espaciotemporal del paisaje sonoro urbano. Una comparación transcontinental entre Venecia (Italia) y Popayán (Colombia) ${ }^{1}$
}

\author{
Spatiotemporal variability of urban soundscape: \\ a cross-continental comparison between Venice (Italy) \\ and Popayán (Colombia)
}

\author{
Julián Grijalba² 주
}

\begin{abstract}
RESUMEN
La provisión de paisajes sonoros sanos y equilibrados es uno de los retos más importantes que afrontan las ciudades del siglo XXI. Actualmente, los estudios relacionados a esta cuestión asumen un enfoque integral, que explora con detenimiento la interacción entre la ciudadanía y su entorno acústico más próximo. El presente trabajo examina el paisaje sonoro de los cascos históricos de Venecia (Italia) y Popayán (Colombia). En ese sentido, se realizaron cartografías del factor físico y factor perceptual del paisaje sonoro urbano, con el propósito de garantizar su eficaz evaluación. Los resultados demuestran patrones de comportamiento espaciotemporal diversos, con un grado de variabilidad que, en esencia, depende del entorno urbano construido. Por tanto, se concluye que el paisaje sonoro debe ser incorporado durante los procesos de planeamiento urbano, de modo que se preste especial atención al balance saludable entre la ecotopofonía y la antropofonía producida en el espacio público abierto de la ciudad. En efecto, este es un ámbito que puede contribuir a la conformación de experiencias sonoras altamente aceptadas por los habitantes urbanos. En suma, se recomienda ampliar la escala de evaluación en futuros estudios y profundizar en la comprensión de la ciudad y su paisaje sonoro resultante.
\end{abstract}

Palabras clave: paisaje sonoro, planeamiento urbano, cartografías sonoras, grabación biaural, ruido urbano

\section{ABSTRACT}

Providing healthy and balanced soundscapes is one of the most important challenges facing cities in the 21st century. Currently, the studies related to this issue assume a comprehensive approach, which carefully explores the interaction between citizens and their closest acoustic environment. This work examines the soundscape of the historic centers of Venice (Italy) and Popayán (Colombia). In this sense, cartographies of

Este texto presenta el resultado de una investigación original apoyada por la Institución Universitaria Colegio Mayor del Cauca, que, en años recientes, ha impulsado con determinación el desarrollo de actividades de ciencia, tecnología e innovación desde los confines de la República de Colombia.

Profesor de la Institución Universitaria Colegio Mayor del Cauca, Centro de Estudios Urbanos, Grupo de Investigación en Diseño y Arte (D\&A), Colombia. Correo electrónico: juliangrijalba@unimayor.edu.co 


\begin{abstract}
the physical factor and perceptual factor of the urban soundscape were carried out, in order to guarantee their effective evaluation. The results show diverse patterns of spatio-temporal behavior, with a degree of variability that, in essence, depends on the built urban environment. Therefore, it is concluded that the soundscape must be incorporated during urban planning processes, so that special attention is paid to the healthy balance between ecotopophony and anthropophony produced in the open public space of the city. Indeed, this is an area that can contribute to the shaping of sound experiences universally accepted by urban inhabitants. In short, it is recommended to expand the evaluation scale in future studies and to deepen the understanding of the city and its resulting soundscape.
\end{abstract}

Keywords: Soundscape, Urban planning, Sound cartographies, Binaural recordings, Urban noise

La contaminación acústica amenaza seriamente la salud pública y perjudica las posibilidades recreativas de los asentamientos humanos (Stanners \& Bourdeau, 1995; OECE, 2003; WHO, 2018). No obstante, se trata de una cuestión que se incorpora débilmente en el planeamiento urbano, con medidas de control que se centran simplemente en la disminución de los niveles de ruido producidos por la ciudad. Ahora bien, a pesar de que estas medidas no siempre son eficientes para mitigar el impacto adverso del ruido en el bienestar social de la población, los debates públicos de muchas ciudades del mundo aún siguen direccionando la mayoría de sus esfuerzos hacia una política enmarcada solo en la reducción del ruido urbano (Raimbault \& Dubois, 2005; Rehan, 2016). Por tanto, diversos estudios argumentan hoy en día la necesidad de recurrir a la ecología acústica para ampliar la comprensión del sonido urbano y contribuir, de esa forma, a la conformación de ciudades con las más altas condiciones de salubridad y habitabilidad posibles (Kang \& Schulte-Fortkamp, 2016).

El concepto de paisaje sonoro se define como «el entorno acústico percibido, experimentado y/o entendido por una o varias personas, en el contexto [en el que se produce] $»^{3}$ (ISO, 2014: 1). Por consiguiente, paisaje sonoro se diferencia de entorno acústico en que: el primero, se refiere a un constructo perceptual; y el segundo, a un fenómeno netamente físico. En ese sentido, considerar la respuesta humana al entorno acústico es primordial en la identificación de los valores positivos de cada paisaje sonoro en particular (Schafer, 1977; Truax, 2006). Al respecto, se resalta que el carácter benéfico de los valores referidos es debido, especialmente, a su la capacidad de evocar pensamientos y emociones, que inciden favorablemente en nuestro estado de ánimo y comportamiento (Southworth, 1969).

Tradicionalmente, el sonido producido por la ciudad se ha catalogado como intrusivo e indeseable. A menudo, los estudios urbanos se aproximan a dicha cuestión de forma parcial y se centran unilateralmente en la evaluación del factor físico (Agnew, 2011). Esta suele efectuarse mediante mapas de ruido que identifican lugares con excesivos niveles de presión sonora, llamados en inglés Sound Pressure Levels (SPL) (Geraghtya \& O'Mahony, 2016; Campello et al., 2017; Vasilyev, 2017; Wang et al., 2018; Di et al., 2018). Ahora bien, más allá de este enfoque, se debe considerar que mejorar la calidad del entorno urbano a través de la reducción de los SPL es poco precisa, puesto que no tiene en cuenta la diferenciación de las diversas clases de sonido percibidas y las posibilidades, de algunas de estas clases, de ocasionar experiencias con alto grado

La traducción es propia del autor, como la de todas las otras citas en lengua distinta al español 
de aceptación por parte de los habitantes urbanos: desencadenar recuerdos agradables, al igual que alentar a la relajación y recuperación, son ejemplos relacionados (Cerwén, 2016; Sun et al., 2019). En ese orden de ideas, es importante destacar que diversas configuraciones urbanas proporcionan paisajes sonoros igualmente diferenciados, que de ser adecuados pueden contribuir convenientemente al bienestar y atractivo de la ciudad (Leus \& Herssen, 2015; Aletta et al., 2019). De ahí, que en la actualidad el paisaje sonoro urbano, referido a la percepción del entorno acústico por parte de la ciudadanía, esté emergiendo como una alternativa a la mirada sesgada y/o reduccionista del ruido urbano (Pijanowski et al., 2011).

Varias investigaciones han evaluado la interacción entre la ciudadanía y su entorno acústico más próximo (Torija et al., 2020; Jia et al., 2020; Jeon \& Jo, 2020; Filipan et al., 2019; Kogan et al., 2018). En tal caso, se reconoce que el carácter de la ciudad obedece, en parte, a la percepción del paisaje sonoro (Farina, 2014; Liu et al., 2019). Liu \& Kang (2016: 32) aseguran que "[h]asta cierto punto, comprender el sonido urbano no depende solo de sus propiedades físicas, sino también de sus atributos positivos o negativos relacionados [con la percepción humana]". Esto se puede notar cuando "dos sonidos con el mismo [SPL] conllevan a sensaciones muy diferentes en el oyente" (Gonzalo et al., 2015: 2). Por ejemplo, los habitantes de la ciudad de Sheffield (Reino Unido) demuestran actitudes opuestas hacia el sonido del tranvía: de un lado, que es desagradable, por ser demasiado monótono y aburrido; y de otro lado, que es muy agradable, por estar relacionado, entre otras cosas, con el pronto regreso a casa (Liu \& Kang, 2016). Por esta razón, y más allá del factor físico que resulta limitante, es preciso abordar el factor perceptual (Cain et al., 2013; Watts \& Pheasant, 2015). Así, un trabajo de paisaje sonoro urbano se considera adecuado si logra englobar todos los factores concernidos (Aletta et al., 2016).

La evaluación del paisaje sonoro consiste en la descripción del entorno acústico, por medio de la identificación de sus fuentes sonoras más relevantes (Raimbault \& Dubois, 2005). En atención a lo anterior, se sugiere clasificar aquellas fuentes de sonido de acuerdo con sus rasgos sonoros más notables, las clases más representativas son: la biofonía, que es igual al conjunto de sonidos biológicos no humanos en un hábitat determinado; la geofonía, conformada por sonidos naturales no biológicos que provienen del ambiente geofísico; y la antropofonía, que, como su nombre lo indica, son aquellos sonidos derivados de acciones realizadas por el hombre (Pijanowski et al., 2011). La taxonomía ha orientado dicha clasificación en función de dos criterios: el primero, que sea aplicable en cualquier entorno acústico; y el segundo, que la terminología de las fuentes sonoras sea seleccionada cuidadosamente, para no incurrir en juicios de valor (Brown et al., 2011). Esta clasificación se puede aplicar fácilmente a grabaciones biaurales en laboratorios de audio o a registros de sonoridad percibida in situ (Oldoni, 2013; Liu et al., 2013a). De igual forma, también cabe considerar rangos de determinación más amplios. Kogan et al. (2017: 2) agregan que «el sonido es un recurso que puede planificarse y gestionarse tanto el espacio como en el tiempo". Por ello, algunas evaluaciones del paisaje sonoro suman también la dimensión espaciotemporal a su desarrollo (Liu et al., 2013b; Hong \& Jeon, 2017).

En Asia, Europa y Estados Unidos se cuentan con diversos estudios sobre paisaje sonoro urbano (Vogiatzis \& Remy, 2014; Romero et al., 2016; Cerwán et al., 2017; Jeon et al., 2018; de la Prida et al., 2019), mientras que en Latinoamérica aún se sigue prestando poca atención investigadora a este respecto. Maristany, López \& Rivera (2016), por ejemplo, llevaron a cabo un trabajo ejemplar al analizar el paisaje sonoro de una red de espacio público en la ciudad de Córdoba (Argentina). 
En esta contribución, se comprobó que los parámetros objetivos y subjetivos del sonido estaban relacionados con la calidad ambiental del lugar. Otra aportación sobresaliente, es la de Szeremeta \& Zannin (2009), quienes examinaron el paisaje sonoro de los parques urbanos en la ciudad de Curitiba (Brasil). El trabajo, a través del análisis físico y perceptivo del sonido, identificó varias condiciones (usos y coberturas del suelo, y tráfico vehicular) que incidieron en la valoración del entorno acústico. Por último, Hermida \& Pavón (2019) evaluaron el paisaje sonoro de las ciudades de Brasilia (Brasil) y Bogotá (Colombia). Esta labor empleó grabaciones biaurales que constataron el nexo entre los atributos perceptivos del sonido urbano y su referenciación en el espacio geográfico.

De acuerdo con el escenario anterior, el presente trabajo plantea un estudio comparativo del paisaje sonoro aplicado a un hecho paradigmático: los cascos históricos de Venecia (Italia) y Popayán (Colombia). El trabajo se enmarca en un proyecto internacional de investigación y creación artística ${ }^{4}$, que explora con detenimiento las percepciones del entorno urbano a través de la pintura y el arte sonoro. En concreto, el aspecto investigativo de dicho proyecto da origen a este artículo y se despliega en dos instancias: la primera, es la evaluación del paisaje sonoro en la ciudad de Popayán, en el año 2017 (Grijalba \& Córdoba, 2020); y la segunda, es la réplica del método obtenido en la primera instancia, pero esta vez ajustado a la ciudad de Venecia, en el año 2019. El proyecto relacionado es, a su vez, parte de una iniciativa universitaria amplia en urbanismo y ordenamiento territorial, que se promueve desde el año 2014 en el suroccidente de Colombia (Córdoba et al., 2016, Morales, 2020; Mazorra et al., 2021).

Así pues, el objetivo definido aquí consiste en examinar la variabilidad espaciotemporal del paisaje sonoro de las áreas antiguas de: (1) Venecia, en la región peninsular del noreste de Italia; y (2) Popayán, en la región andina más suroccidental de Colombia. En esencia, la selección de estas ciudades radica en que sus determinantes contextuales diferenciadas, son el punto de partida para entrever la conformación de paisajes sonoros con igual grado de divergencia y pluralidad. En tal sentido, de entrada, se describen las consideraciones metodológicas tenidas en cuenta para la obtención de resultados, que procede a partir de una descripción contextual de las áreas de estudio. El apartado siguiente, se despliega sobre todo en forma de cartografías sonoras, comprendidas entre el factor físico y el factor perceptual del paisaje sonoro. Finalmente, y conforme al análisis expuesto, se discute sobre la importancia del paisaje sonoro en el planeamiento urbano, que conlleva a una serie de conclusiones y propuestas sobre futuras líneas de investigación en la materia.

\section{Método}

\section{Áreas de estudio}

El análisis se llevó a cabo en las áreas históricas de Venecia (Italia) y Popayán (Colombia) (Figura $\mathrm{N}^{\circ}$ 1a-b). En el caso de Venecia, el área de estudio seleccionada es su casco histórico,

El proyecto titulado "La abstracción sintética del paisaje sonoro urbano. Un estudio en las ciudades de Venecia (Italia) y Popayán (Colombia)" (2016-2020), fue una colaboración de la artista conceptual Ivana Blanco Gross, el músico compositor Steve Layton y el arquitecto Julián Grijalba. 
reconocido en la lista del Patrimonio Mundial de la UNESCO en 1987 (WCED, 1987). Tal sector se localiza al noreste del mar Adriático sobre un conjunto único de islas en una laguna costera y se encuentra definido por el Plan de Planificación Territorial del Ayuntamiento de Venecia (2014). De acuerdo con este plan, el área histórica veneciana se conforma por seis distritos, a saber: Castello, San Marco, San Polo, Dorsoduro, Santa Croce y Cannaregio, dentro de los cuales se incluyen las de islas de Tronchetto, Sacca San Biagio, Sacca Fisola, Giudecca, San Giorgio y Sant'Elena (Figura $\left.N^{\circ} 1 a\right)$. El paisaje urbano del área mencionada presenta el carácter representativo de una ciudad europea del Medioevo, con un entramado urbano irregular y sin aparente orden. Dicha morfología está dada, principalmente, por los tradicionales campos 5 italianos, que, en Venecia, se transformaron en verdaderas plazas públicas y que terminaron interconectándose a través de cientos de calles y puentes peatonales, así como también mediante decenas de canales acuáticos.

En general, el sector estudiado se encuentra rodeado de edificios monumentales (sobre todo, monasterios, palacios e iglesias), que preservan bastante bien los estilos arquitectónicos más influyentes de la historia occidental (bizantino, gótico, renacentista, entre otros). Además de su valoración patrimonial, la consideración más destacable es que la ciudad histórica de Venecia es ampliamente conocida por su atractivo turístico internacional y por ser uno de los puertos de cruceros más activos del sur de Europa, con alrededor de 60.000 visitantes por día (Modak, 2017) y una población local de tan solo 52.143 habitantes (Comune di Venezia, 2019). Otra condición sobresaliente es que el tránsito vehicular de esta zona se encuentra restringido al medio lagunar y funciona a través de una red pública de canales acuáticos en la que navegan embarcaciones de diferentes envergaduras, entre los canales más notables se encuentran: Río Novo, el canal de la Giudecca, el canal de Cannaregio y el Gran Canal. En las intermediaciones del último canal mencionado se localiza el emblemático puente de Rialto, que conecta a San Polo con San Marco y que reúne una gran cantidad de mercados locales y tiendas minoristas en sus alrededores. En adición, se tiene que el único acceso terrestre a la ciudad está supeditado a la estación ferroviaria de Santa Lucía y a la estación de buses de Piazzale Roma.

En el caso de Popayán, el área de estudio delimitada es el sector central de su casco histórico, el cual concentra el mayor valor patrimonial de la ciudad. Se define de acuerdo con el Plan Especial de Manejo y Protección (PEMP), elaborado por la Alcaldía del Municipio de Popayán (2010). A partir del cual, se escoge el área denomina «unidad de gestión urbanística de Santo Domingo», conformada por dos a tres cuadras en torno a la típica plaza central colonial hispanoamericana, que, en el caso de Popayán, se convirtió en el actual Parque Francisco José de Caldas (Alcaldía del Municipio de Popayán, 2010) (Figura Nº1b). El área limita al norte con el río Molino, en cuyo margen se localizan los parques José Hilario López y Arcada de la Herrería. En general, el sector estudiado refleja el patrón esperable de una ciudad colonial hispanoamericana, no solo por la trama ortogonal, sino por las construcciones de bahareque de planta baja más una sola altura (dos o más excepcionalmente), que ocupan las fachadas de todas las manzanas adyacentes.

De acuerdo con el último recuento disponible, la población del área de estudio payanesa es de 1.783 habitantes (DANE, 2018). Ahora bien, se debe considerar que se hace referencia al área central de Popayán, con funciones comerciales, de servicios especializados y administrativos de primer rango, por lo que el área actúa como un polo de atracción que reúne a diario a toda la po- 
blación urbana; estimada en 267.389 habitantes (DANE, 2018). Al mismo tiempo, el turismo nacional e internacional de Popayán también se concentra aquí, dado su indiscutible valor patrimonial, tanto material (paisaje urbano, catedral, claustros, iglesias, etc.) como inmaterial (semana santa y congreso gastronómico) (UNESCO, 2007; UNESCO, 2009).

Figura $\mathrm{N}^{\circ} 1 \mathrm{a}-\mathrm{b}$.

Áreas de estudio: casco histórico de Venecia, en la parte superior (a), y sector central del casco histórico de Popayán, en la parte inferior (b).


Fuente: Elaboración propia. 


\section{Datos recabados}

Los datos del área de estudio de Venecia se recolectaron en febrero de 2019 y los del área de estudio de Popayán en octubre de 2017. La recolección de estos datos estuvo determinada por dos aspectos fundamentales: el primer aspecto, fue la asignación de mallas de muestreo para asegurar la evaluación de los principales factores del paisaje sonoro urbano, por lo que se siguió a Wei et al. (2016), en cuanto al factor físico, y a Liu et al. (2013b), en lo relacionado con el factor perceptual; y el segundo aspecto, fue el ajuste de la taxonomía propuesta por Brown, Kang \& Gjestland (2011), con el fin de definir las clases de sonidos evaluadas en este trabajo (Figura $\mathrm{N}^{\circ} 2$ ).

Figura $\mathrm{N}^{\circ} 2$.

Taxonomía del paisaje sonoro ajustada a Venecia y Popayán

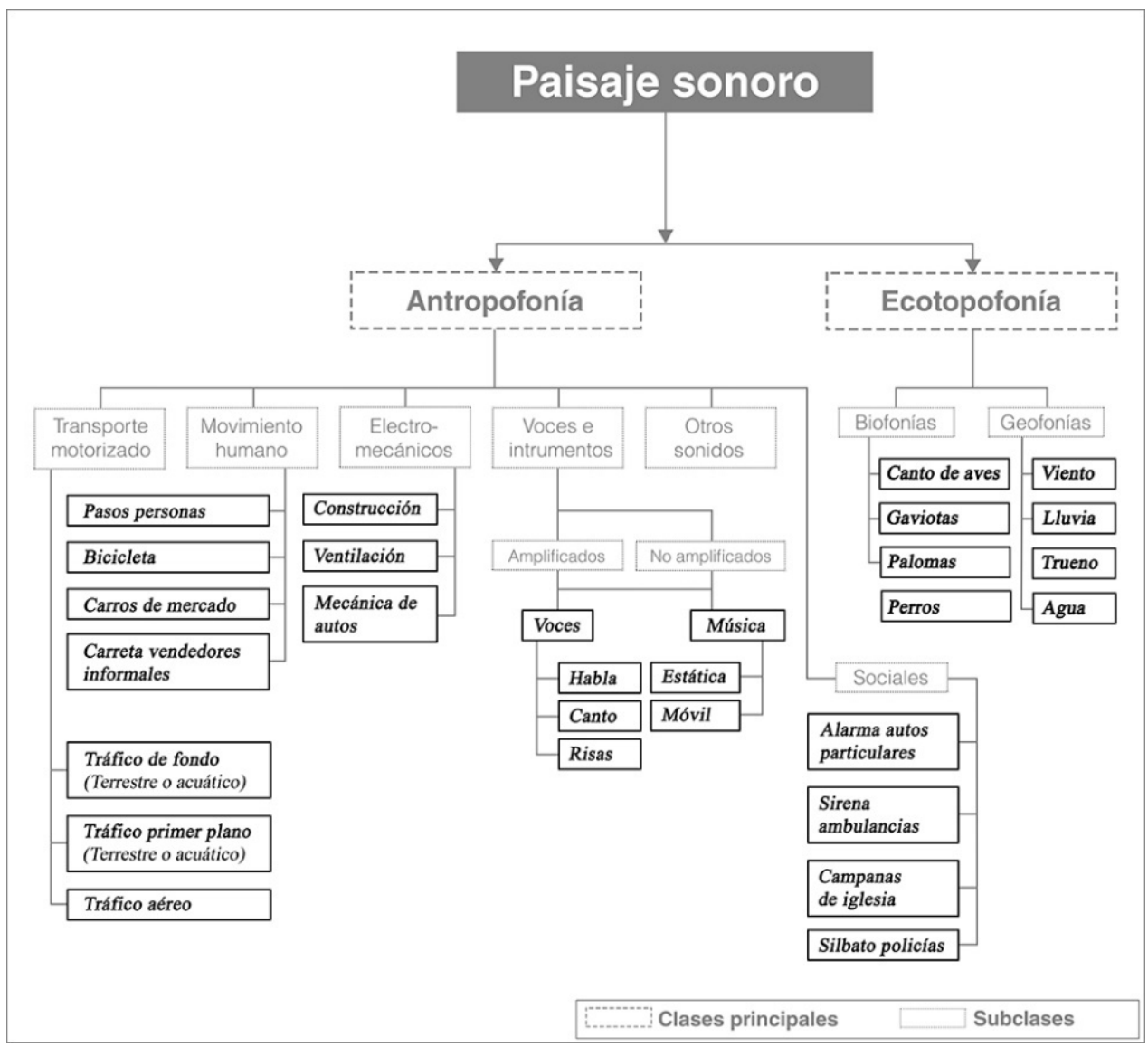

Elaboración del autor a partir de Brown, Kang \& Gjestland (2011).

En el área de estudio de Venecia, se asignaron dos mallas de $200 \times 200$ m, con distribuciones de puntos similares: la primera, 'malla MV1', con 106 puntos; y la segunda, 'malla MV2', con 103 puntos (Figura $\mathrm{N}^{\circ} 3$ ). El tiempo de registro en estas mallas se fijó en 3 minutos por cada punto de muestreo y los datos tanto físicos como perceptuales se tomaron de forma simultánea. En relación con el factor físico, se optó por la malla MV1 (Figura Nº3MV1) y se realizaron mediciones 
acústicas a una distancia de 1,5 m del suelo con un sonómetro Sound Pro DL Clase II de bandas de $1 / 3$ de octava. Las mediciones se verificaron por medio de un calibrador de alta precisión AcoustiCal AC-300. Los indicadores acústicos evaluados fueron: el nivel mínimo, el nivel equivalente y el nivel máximo de presión sonora $\left(\mathrm{dB}_{\text {Min' }} \mathrm{dB}_{\text {Leq, }} \mathrm{dB}_{\mathrm{Max}}\right)$, durante horario diurno (8:00-18:00). De correspondencia al factor perceptual, se utilizó tanto la malla MV1 como la malla MV2, en estas se realizaron grabaciones biaurales asistidas por micrófonos SONY ECM-XM1 y una grabadora TASCAM DR-40. Posteriormente, en un laboratorio de audio y con base en la taxonomía ajustada, la sonoridad percibida se registró por un grupo de diez examinadores sin deficiencias auditivas (todos ellos estudiantes de arquitectura), quienes se entrenaron previamente para realizar la identificación de los diferentes sonidos.

Figura №3.

Distribución espacial de los puntos de registro en el área de estudio de Venecia.
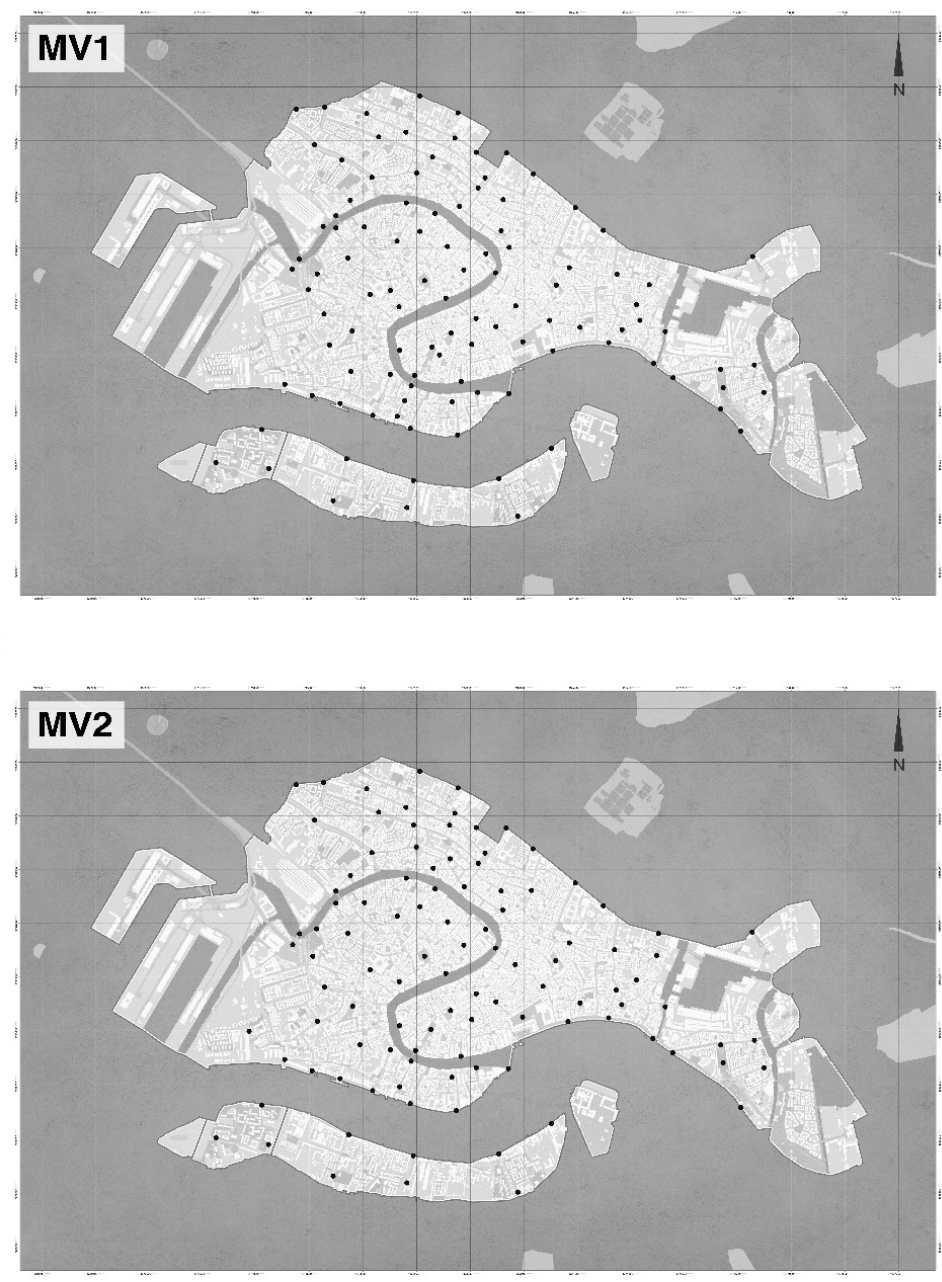

- Puntos de niveles de presión sonora y sonoridad percibida

Fuente: Elaboración propia. 
Los datos perceptuales de Venecia se capturaron en dos períodos sucesivos por cada punto de registro: $1^{\circ}$ período (P1): 8:00-18:00, recolectado en la malla MV1 (Figura $\mathrm{N}^{\circ} 3 \mathrm{MV} 1$ ); $2^{\circ}$ período (P2): 18:00-00:00, recolectado en la malla MV2 (Figura N³MV2). De forma aleatoria, para cada período se realizó el respectivo registro mediante el grupo de examinadores y las grabaciones efectuadas. Estos registros se segmentaron en seis etapas de tiempo secuenciales con duración de 30 segundos cada una, a través de las cuales se realizó la calificación de cada sonido de subclase. Para su determinación, se utilizó una escala lineal de cinco puntos (1=muy bajo; 2=bajo; 3=normal; 4=alto; 5=muy alto). La sonoridad percibida de las subclases del paisaje sonoro, en cada punto de muestreo y período dado, se calculó sumando las calificaciones obtenidas en las seis etapas de tiempo (30 segundos), en las cuales fue dividido cada registro ( 3 minutos). Posteriormente, se asumió que la sonoridad percibida de cada clase principal del paisaje sonoro, en Venecia, fue igual a la suma de las calificaciones de sus respectivas subclases en P1.

En el área de estudio de Popayán, se asignaron las siguientes mallas: una 'malla MP1' de 50 x 50 m, distribuida en 121 puntos; y una 'malla MP2' de 100 x 100, distribuida en 50 puntos (Figura N4). Para el factor físico, se optó por la malla MP1 (Figura N4MP1) y se tomaron mediciones acústicas en horario diurno (8:00-18:00), con una duración de 15 minutos por cada punto. Las demás condiciones de medición fueron idénticas a las descritas en el caso de Venecia. En cuanto al factor perceptual, se optó por la malla MP2 (Figura N4MP2) y, de nuevo, a partir de la taxonomía ajustada, se registró la sonoridad percibida, esta vez, in situ, por un grupo de seis observadores (cinco estudiantes de arquitectura y un músico), entrenados con anterioridad y sin deficiencias auditivas.

Los datos perceptuales de Popayán se recogieron en cinco períodos sucesivos de dos horas de duración por cada punto de registro: $1^{\circ}$ período (P1): 8:00-10:00; $2^{\circ}$ período (P2): 10:00-12:00; $3^{\circ}$ período (P3): 12:00-14:00; $4^{\circ}$ período (P4): 14:00-16:00; $5^{\circ}$ período (P5): 16:00-18:00. Durante cada período se registraron 10 minutos aleatoriamente. El calculó de la sonoridad percibida de cada sonido de subclase en esos registros, se basó en los mismos parámetros aplicados en Venecia, con la salvedad de que en este caso se calificaron un total de veinte etapas de tiempo, ya que el tiempo registrado aquí, fue más prolongado (10 minutos). Por último, la sonoridad percibida de cada clase principal del paisaje sonoro, en Popayán, fue la suma de las calificaciones de sus respectivas subclases durante todos los períodos de registro. 
Figura $\mathrm{N}^{\circ} 4$.

Distribución espacial de los puntos de registro en el área de estudio de Popayán.

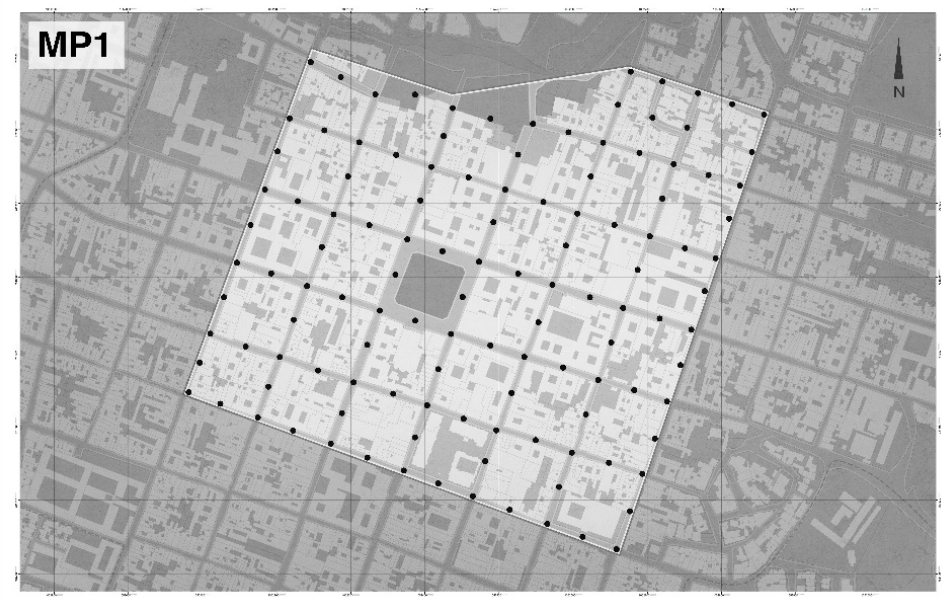

- Puntos de niveles de presión sonora

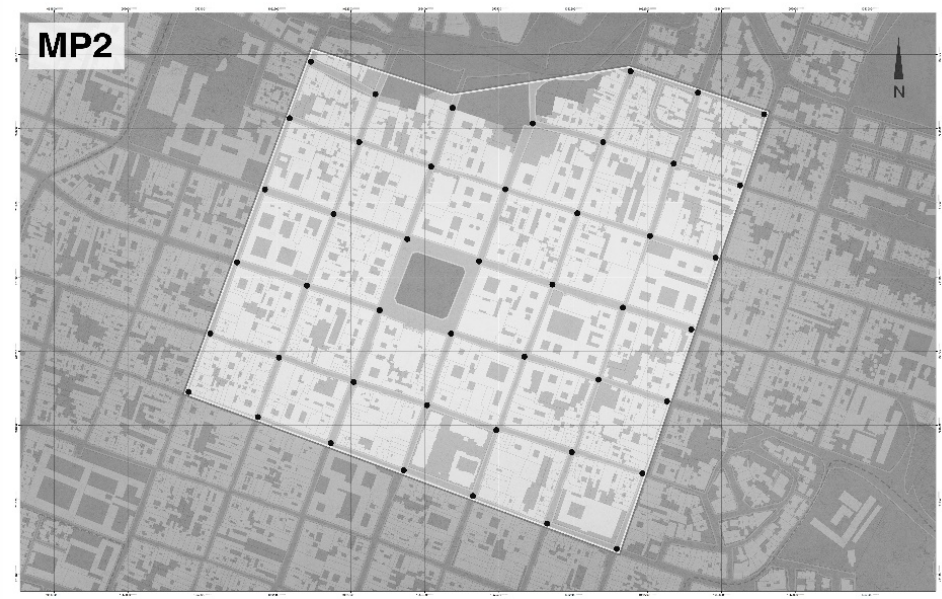

- Puntos de sonoridad percibida

Fuente: Elaboración propia.

\section{Tratamiento geoestadístico}

Una vez clasificados los indicadores acústicos (factor físico) y las calificaciones de sonoridad percibida (factor perceptual) como datos de análisis, se efectuó el correspondiente tratamiento geoestadístico. Para tal fin, se siguió el método de regionalización de las variables estudiado por Harman, Koseoglu y Yigit (2016), particularmente el de interpolación espacial "krigeaje" (kriging en inglés). Este método se seleccionó debido a su exactitud en la predicción de datos espaciales. La fórmula del "krigeaje" es:

$$
N_{o}=\sum_{i=1}^{n} W_{i} N_{i}
$$


Fórmula en la que, $N_{0}$ representa el valor de la variable del paisaje sonoro a interpolar en el punto $\left(x_{o^{\prime}} y_{0}\right)$ y $W_{i}$ son los pesos que corresponden a cada $N_{i}$ en $\left(x_{i}, y_{i}\right)$, usados en el cálculo de $N_{o}$. $N_{i}$ corresponde a los valores de los puntos de referencia empleados en el cálculo y $n$ representa el número total de las variables utilizadas. Para su resolución, se calcularon modelos de semivariancia experimental por cada una de las variables del paisaje sonoro. Luego los modelos de semivariancia se ajustaron a los modelos teóricos del krigeaje: exponencial, gaussiano y esférico, a través de lo cual se seleccionaron los más adecuados para la interpolación de cada variable. De ese modo, se aseguró la creación de las superficies de interpolación, necesarias para su posterior mapeo (Goovaerts, 1999). Todos los cálculos mencionados se llevaron a cabo en el programa RStudio.

\section{Mapeo del paisaje sonoro}

El mapeo del paisaje sonoro urbano consiste en la visualización de su patrón de comportamiento espaciotemporal. Los mapas se elaboraron en el sistema de información geográfica ArcMap 10.3.1 y Adobe Photoshop CC 2018, a partir de las superficies de interpolación procesadas en el tratamiento geoestadístico. El presente trabajo elige cartografiar tanto el factor físico como el perceptual del paisaje sonoro, aunque usualmente se limite solo a uno de los dos. La cartografía elaborada estructura el apartado de resultados y se presenta a continuación.

\section{Resultados}

\section{Factor físico}

Los mapas de resultados demuestran que el factor físico del paisaje sonoro presenta indicadores acústicos con concentraciones espaciales similares en cada área de estudio, pero diferenciada entre cada una de ellas (Figura $\mathrm{N}^{\circ} 5$ y $\mathrm{N}^{\circ} 6$ ). En Venecia, los niveles más bajos se registran en las plazas y parques públicos con baja afluencia de visitantes al interior de los diferentes distritos venecianos, mientras que incrementan al acercarse a los canales de mayor envergadura y a la zona de servicio de transporte público terrestre. Los niveles incrementan también en plazas públicas de mayor afluencia peatonal, debido a su connotación turística especial, como sucede en la Piazza San Marco. En todo caso, los niveles más altos tienden de forma general a reunirse: al noroeste, en la estación de autobuses de Piazzalle Roma, en la estación ferroviaria de Santa Lucía y en el canal de Cannaregio; y, al sur y sudeste, sobre los canales de la Giudecca y Galeazze, respectivamente. Por otro lado, en Popayán, los niveles más bajos se registran en el Parque Caldas, en el centro, y en los parques del río Molino, en el sector septentrional, en cambio, en los demás márgenes del área de estudio payanesa aumentan como consecuencia de la actividad comercial y del intenso flujo vehicular.

Los indicadores acústicos presentan rangos diferenciados y se representan con una gama cromática similar, que empieza en verdes claros y oscuros (valores bajos), luego cambia a naranjas y rojos (valores intermedios), y termina en tonos magentas y azulados (valores altos). Acorde con los registros de Venecia, los niveles mínimos se encuentran entre 38 a $59 \mathrm{~dB}_{\text {Min }}$ (Figura $\mathrm{N}^{\circ} 5 \mathrm{a}$ ); los niveles equivalentes oscilan entre 48 a $73 \mathrm{~dB}_{\text {Leq }}$ (Figura $\mathrm{N}^{\circ} 5 \mathrm{~b}$ ); y los niveles máximos, entre 60 a 93 $\mathrm{dB}_{\text {Max }}$ (Figura $\mathrm{N}^{\circ} \mathrm{C}$ ). Con relación a los registros de Popayán, los niveles mínimos se hallan entre 
40 a $65 \mathrm{~dB}_{\text {Min }}$ (Figura $\mathrm{N}^{\circ} 6 \mathrm{6}$ ); los niveles equivalentes se fijan entre 52 a $77 \mathrm{~dB}_{\text {Leq }}$ (Figura $\mathrm{N}^{\circ} 6 \mathrm{~b}$ ); y los niveles máximos, entre 60 a $93 \mathrm{~dB}_{\text {Max }}$ (Figura $N^{\circ} 6 \mathrm{c}$ ). En general, los SPL en Popayán superaron a Venecia en $4 \mathrm{~dB}_{\text {Leq }}$.

Figura $\mathrm{N}^{\circ} 5$.

Mapas del factor físico del paisaje sonoro en el área de estudio de Venecia
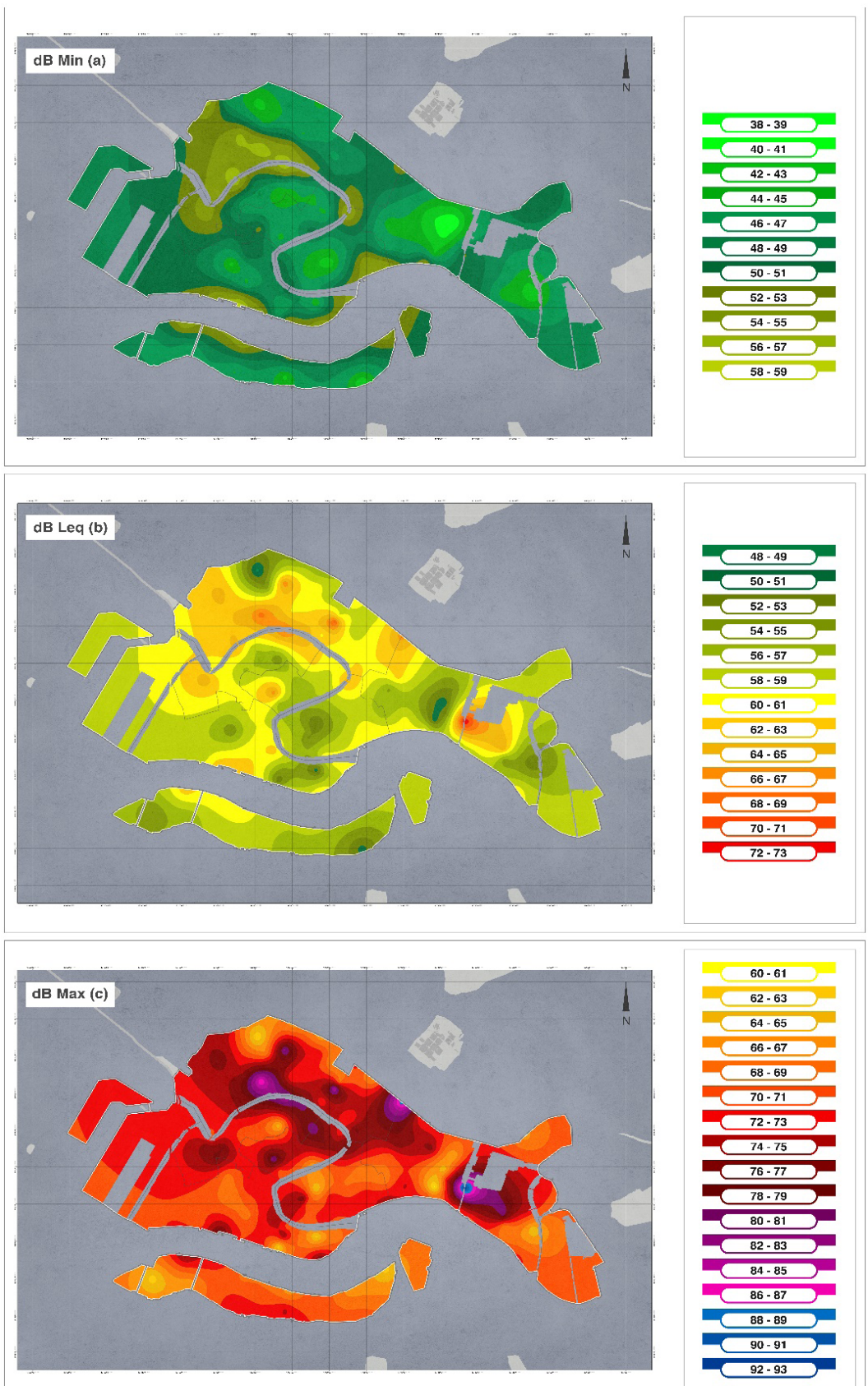

Fuente: Elaboración propia. 
Figura $\mathrm{N}^{\circ} 6$.

Mapas del factor físico del paisaje sonoro en el área de estudio de Popayán
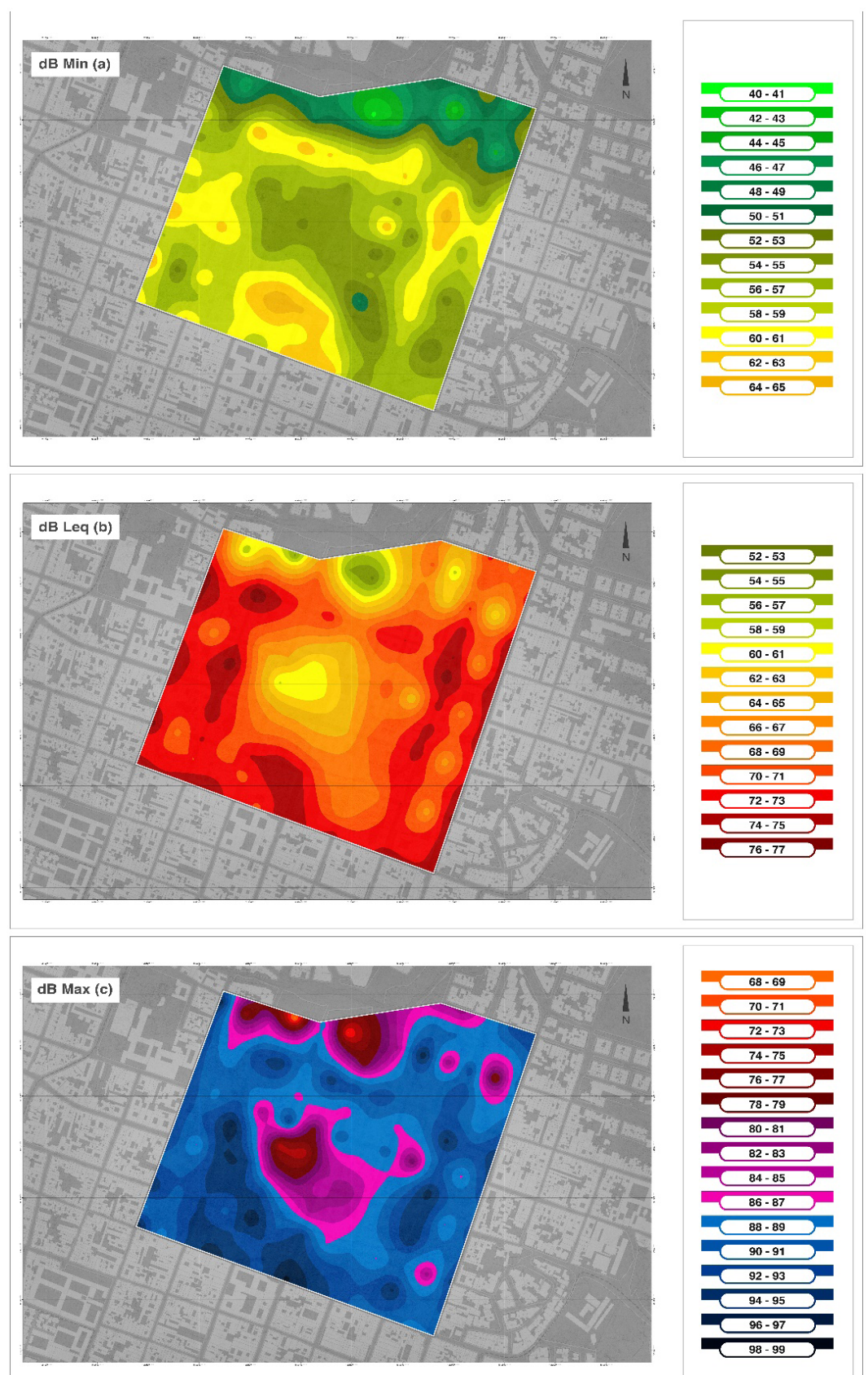

Fuente: Elaboración propia. 


\section{Factor perceptual}

El patrón de comportamiento espacial del factor perceptual del paisaje sonoro se describe en los mapas de clase principal de antropofonía y ecotopofonía, tal y como se muestra en la Figura $\mathrm{N}^{\circ} 7$. En Venecia, la antropofonía se concentró en los distritos de Santa Croce, San Polo y Cannaregio. Este patrón se debe al transporte terrestre y acuático, y al uso del espacio público de esos distritos: hacia el este, se trata de la estación de buses de Piazzale Roma (en Santa Croce) y de la estación ferroviaria de Santa Lucía (en Cannaregio), que son la única vía de acceso terrestre al casco histórico; sobre el límite entre Santa Croce y San Polo, la presencia de peatones que recorren las distintas plazas y mercados públicos del sector, debido a su atractivo comercial y turístico excepcional; y por último, al margen meridional de Cannaregio, el tránsito masivo de personas en diferentes direcciones, así como también la navegación frecuente de embarcaciones sobre el Gran Canal y el canal de Cannaregio (Figura Nº7V-A). En contraste, la ecotopofonía se situó en las áreas verdes de Sacca Fisola y al noroeste de Cannaregio, debido, principalmente, al sonido predominante de la laguna y de las aves acuáticas que rondan en sus alrededores (Figura Nº $7 \mathrm{~V}$-E).

En Popayán, la antropofonía obtuvo mayor grado de calificación en los márgenes oriental y occidental de su área de estudio. La razón de este patrón es el alto flujo de transporte público y privado de esas zonas: hacia el este, se hace referencia al eje de la Carrera 3, que es la vía de paso del casco histórico utilizada por muchas líneas de buses en dirección norte; hacia el noroeste, la principal entrada a la zona antigua en dirección sur, que coincide, además, con el tramo de mayor pendiente en el que el tráfico vehicular acelera para salvar la inclinación de la vía (Figura №7P-A). Por otro lado, la ecotopofonía se concentra en el Parque Caldas y en la ribera del río Molino, a causa, sobre todo, del canto de las aves en esos lugares (Figura $\mathrm{N}^{\circ} 7 \mathrm{P}-\mathrm{E}$ ). 
Figura $\mathrm{N}^{\circ} 7$.

Mapas de antropofonía y ecotopofonía.


Fuente: Elaboración propia.

La Figura $\mathrm{N}^{\circ} 8$ presenta los elementos del entorno urbano que contribuyen a la conformación del sonido antropofónico. Al mismo tiempo, las Figuras $N^{\circ} 9$ y 10 muestran los mapas de subclase de antropofonía en las áreas de estudio. Para el caso de Venecia, los mapas de voces e instrumentos (Figura $\mathrm{N}^{\circ} \mathrm{VVI}$ ) y movimiento humano (Figura $\mathrm{N}^{\circ} \mathrm{gMH}$ ) demuestran un patrón espacial bastante parecido a su clase principal. Juntos dominan la impresión global del paisaje sonoro de antropofonía en un $65,1 \%$ (VI: 38,2\% y MH: 26,9\%). Los demás mapas de subclase presentan patrones 
espaciales diversos, que de cualquier forma aportan en menor medida a la configuración global del paisaje sonoro: transporte motorizado: 25,8\% (Figura N9TM); sociales: 3,8\% (Figura $\mathrm{N}^{\circ} 9 \mathrm{~S}$ ); electromecánicos: 2,9\% (Figura NºEM); otros sonidos: 2,1\% (Figura NºOS).

Para el caso de Popayán, el mapa de subclase de trasporte motorizado es el de mayor similitud a su clase principal (Figura $\mathrm{N}^{\circ} 10 \mathrm{TM}$ ). En efecto, dicha subclase domina la impresión global del paisaje sonoro de antropofonía en un $63,3 \%$. El resto de los mapas de subclase muestran patrones espaciales diferenciados, con dominancias menores: voces e instrumentos: 22,9\% (Figura $\mathrm{N}^{\circ} 10 \mathrm{VI}$ ); movimiento humano: 7,6\% (Figura $\mathrm{N}^{\circ} 10 \mathrm{MH}$ ); sociales: 3,4\% (Figura $\mathrm{N}^{\circ} 10 \mathrm{~S}$ ); sonidos electromecánicos: 2,0\% (Figura $\mathrm{N}^{\circ} 10 \mathrm{EM}$ ); y otros sonidos, 0,5\% (Figura №100S).

Figura $\mathrm{N}^{\circ} 8$

Elementos que originan la producción de antropofonía en las áreas de estudio.
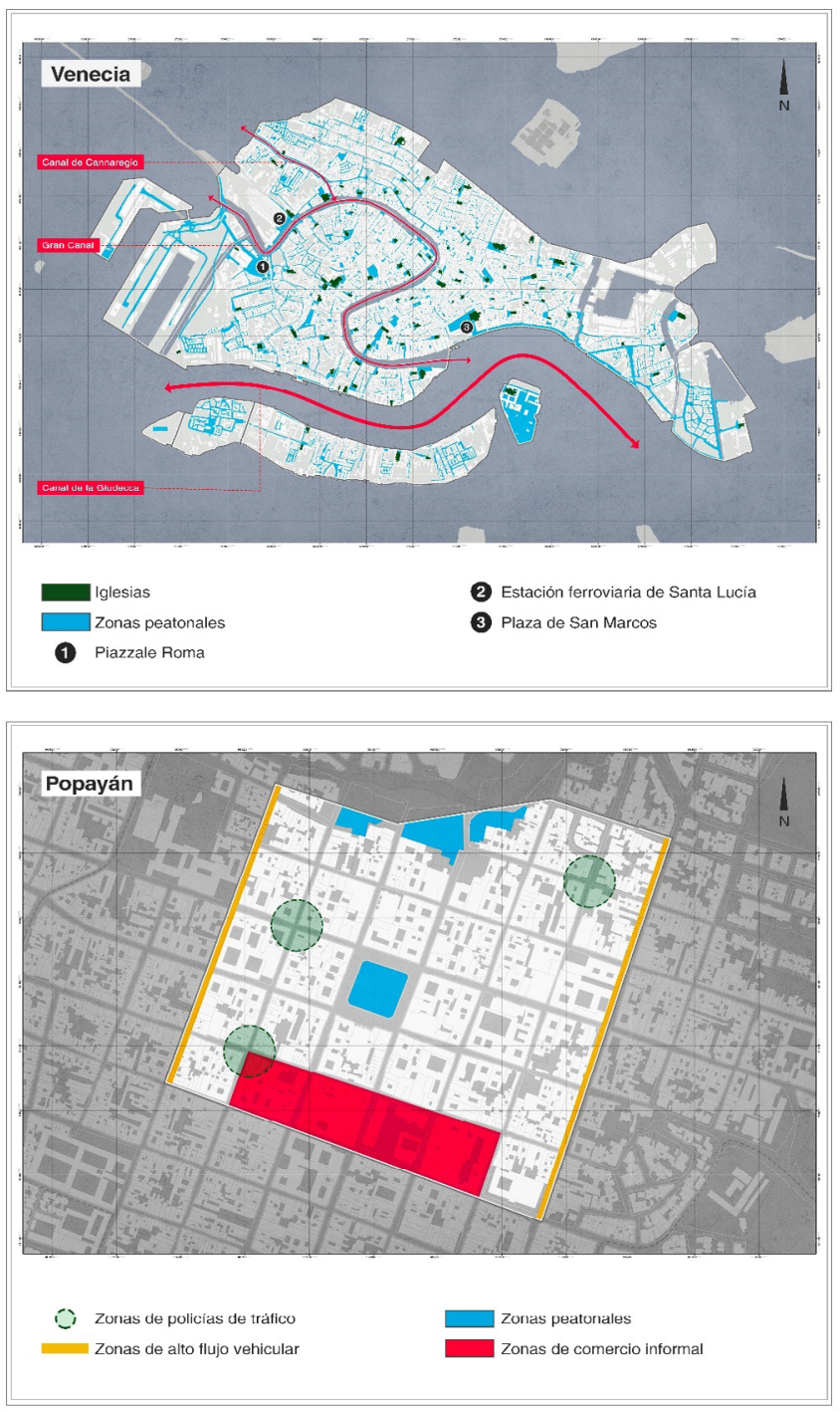

Fuente: Elaboración propia. 
No se observa correlación espacial entre las subclases de menor dominancia de antropofonía en las áreas de estudio. En Venecia, los patrones expresados dependen de varias razones, estas son: TM, la navegación en el canal de la Giudecca, al norte del Gran Canal y en el margen más septentrional de Cannaregio y Castello; S, las campanas de las iglesias y, eventualmente, las sirenas de los servicios de emergencia en Castello y Cannaregio; EM, obras de mantenimiento en espacio público; y OS, causas varias. Igualmente, en Popayán, los patrones están sujetos a las siguientes determinantes: VI, las zonas de mayor concentración de comercio informal en la vía pública; $\mathrm{MH}$, las zonas peatonales del Parque Caldas y de la ribera del río Molino; S, el uso de silbatos por agentes de tránsito en intersecciones viarias; EM, obras civiles en el momento del registro; y OS, motivos diversos.

Figura $\mathrm{N}^{\circ} 9$.

Mapas de subclase de antropofonía en el área de estudio de Venecia.

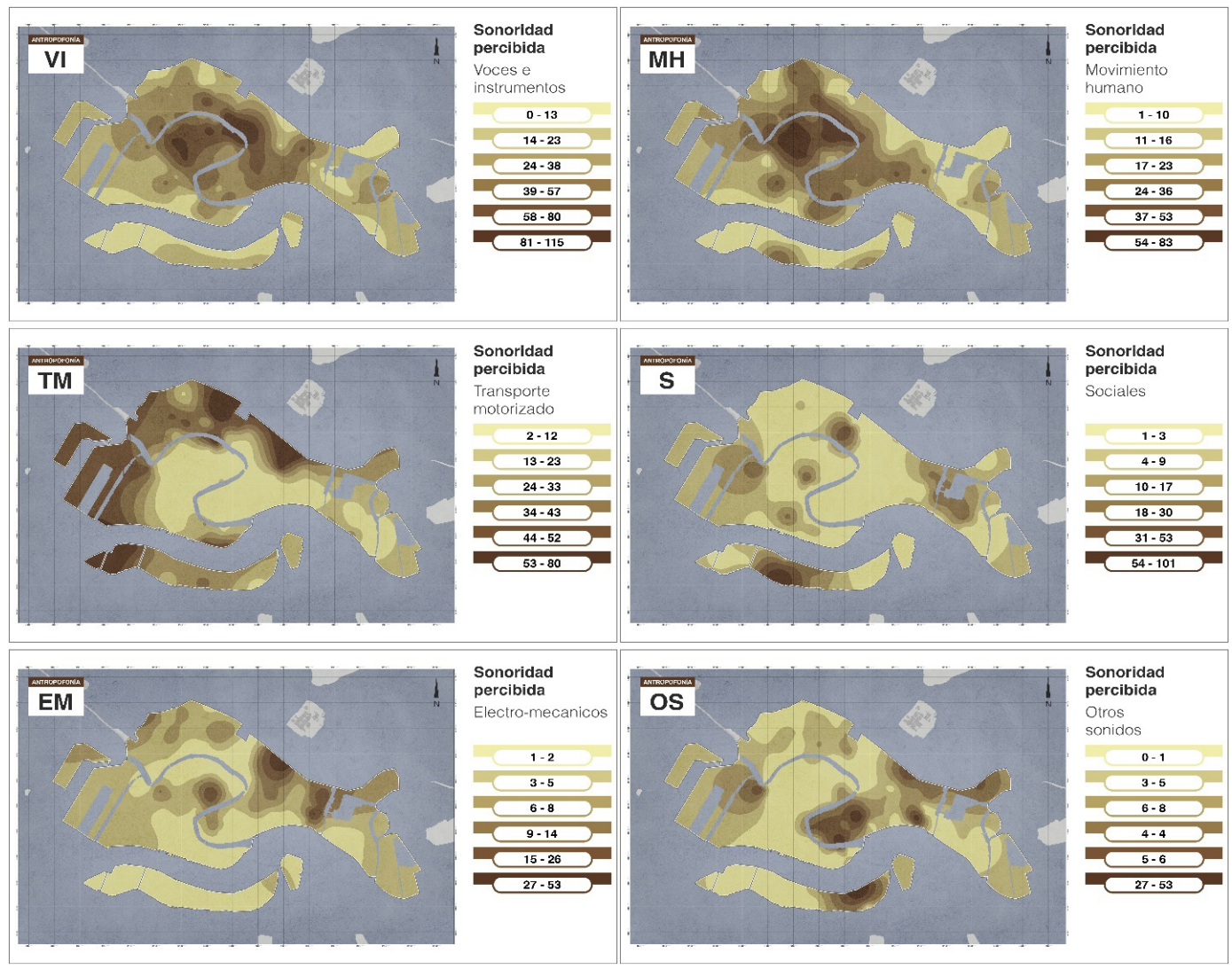

Fuente: Elaboración propia. 
Figura $\mathrm{N}^{\circ} 10$.

Mapas de subclase de antropofonía en el área de estudio de Popayán.

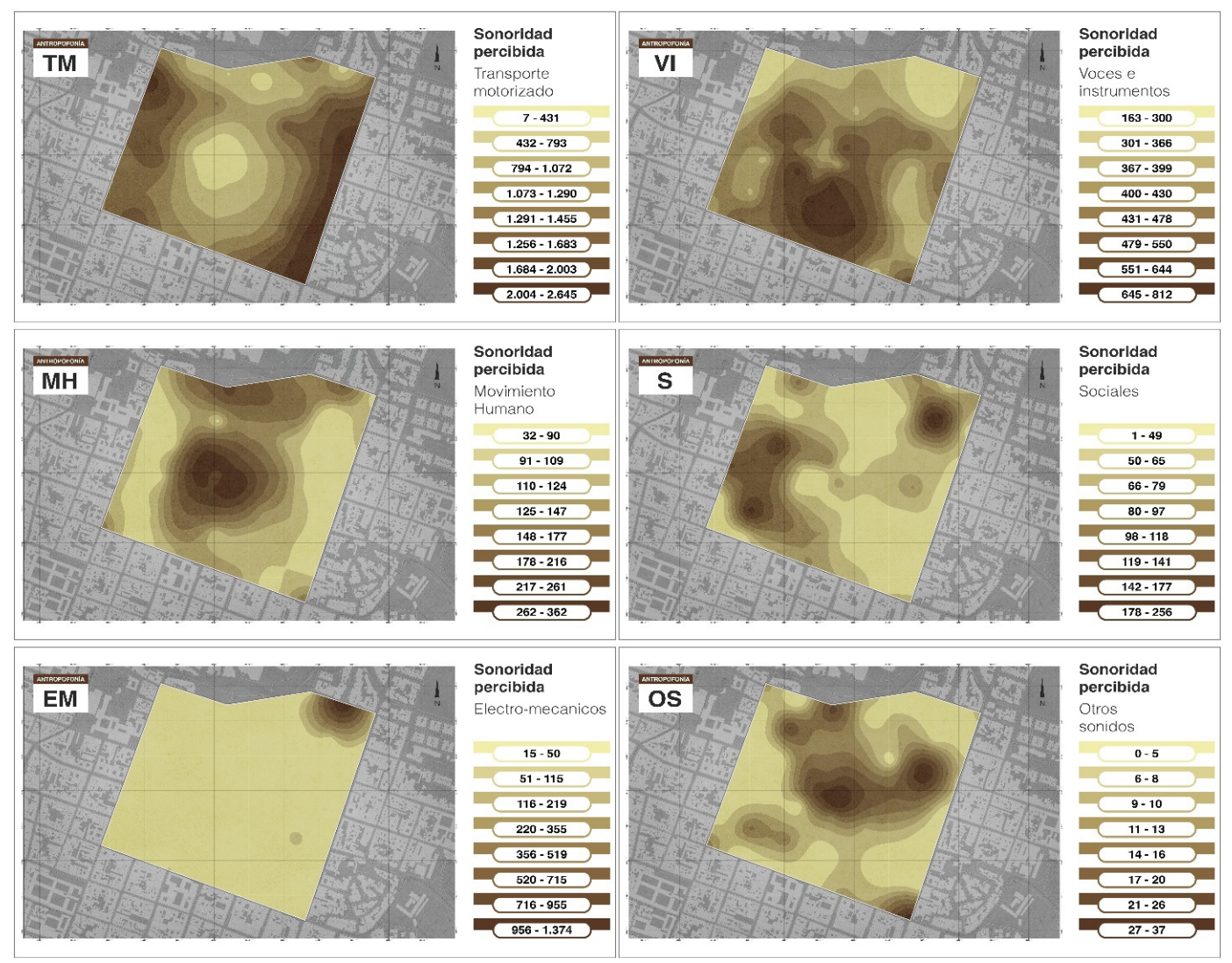

Fuente: Elaboración propia.

A su vez, la Figura $N^{\circ} 11$ delimita los elementos del entorno urbano que generan la concentración del sonido ecotopofónico. Los mapas de subclase de ecotopofonía se exponen en las Figuras $N^{\circ} 12$ y 13 en las áreas de estudio. En Venecia, el mapa de subclase de geofonía (Figura $N^{\circ} 12 G$ ) producido por el agua de la laguna es similar al de su clase principal. Por este motivo, la geofonía domina la impresión global de ecotopofonía (en un 75,8\%). En paralelo, el mapa de biofonía (Figura $\mathrm{N}^{\circ} 12 \mathrm{~B}$ ) muestra un patrón de comportamiento espacial disperso, producido por las gaviotas en la isla de Sacca Fisola y en el noroeste de Cannaregio, y, en algunos casos, por el paseo de mascotas domésticas (perros, sobre todo). 
Figura $N^{\circ} 11$.

Elementos que originan la producción de ecotopofonía
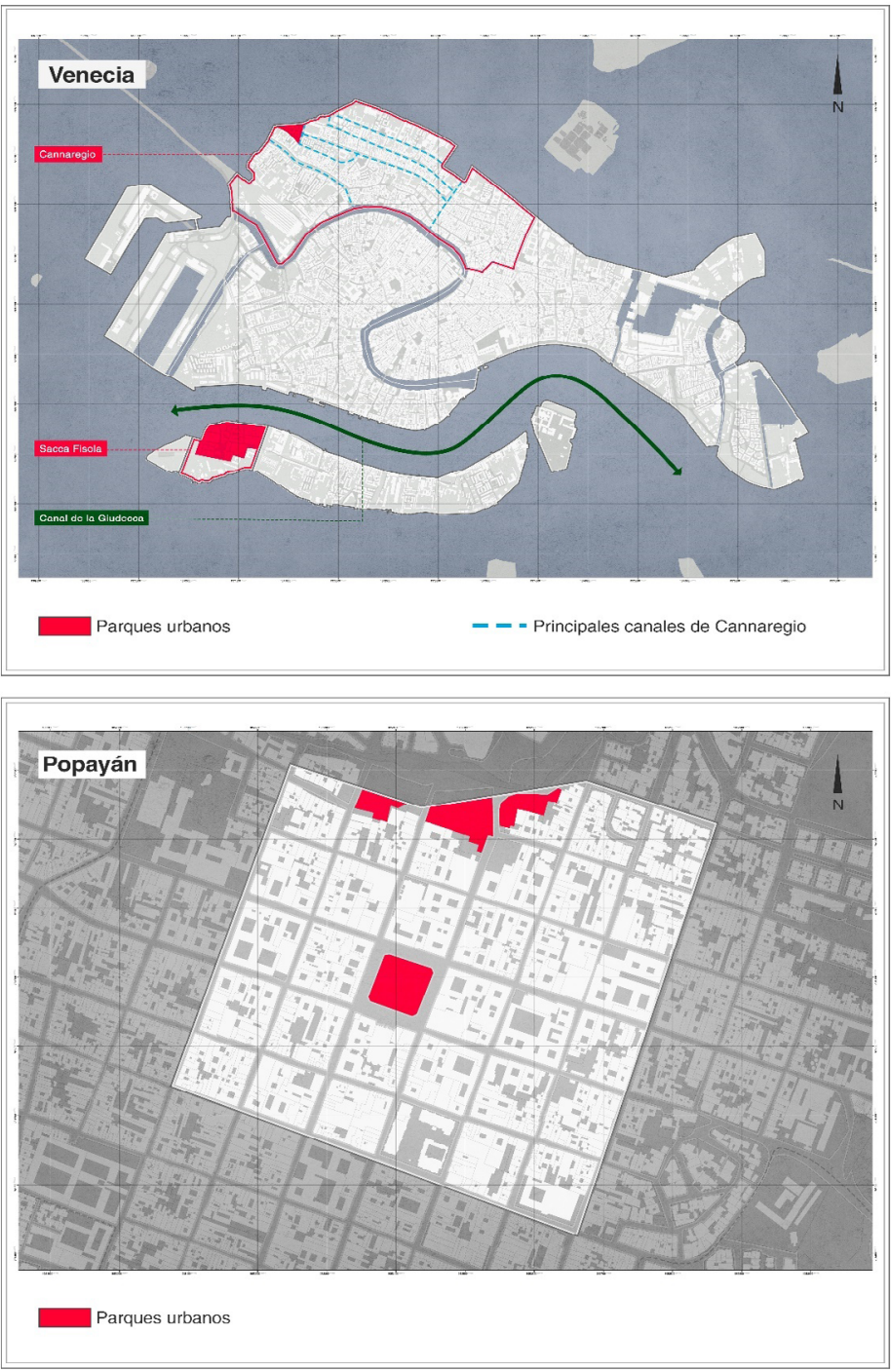

Fuente: Elaboración propia.

En Popayán, el mapa de subclase de biofonía (Figura N¹3B) generado por el canto de las aves demuestra un patrón espacial semejante al de su clase principal, de lo que se infiere que domina la impresión global del paisaje sonoro de ecotopofonía (en un 83,3\%). En contraposición, el mapa de geofonía (Figura $\mathrm{N}^{\circ} 13 \mathrm{G}$ ) presenta un patrón de comportamiento espacial disperso, producido por la lluvia y el viento, que reflejan la manifestación homogénea del clima tropical en el área de estudio. 
Figura $\mathrm{N}^{\circ} 12$

Mapas de subclase de ecotopofonía en el área de estudio de Venecia



Fuente: Elaboración propia.

Figura $\mathrm{N}^{\circ} 13$.

Mapas de subclase de ecotopofonía en el área de estudio de Popayán

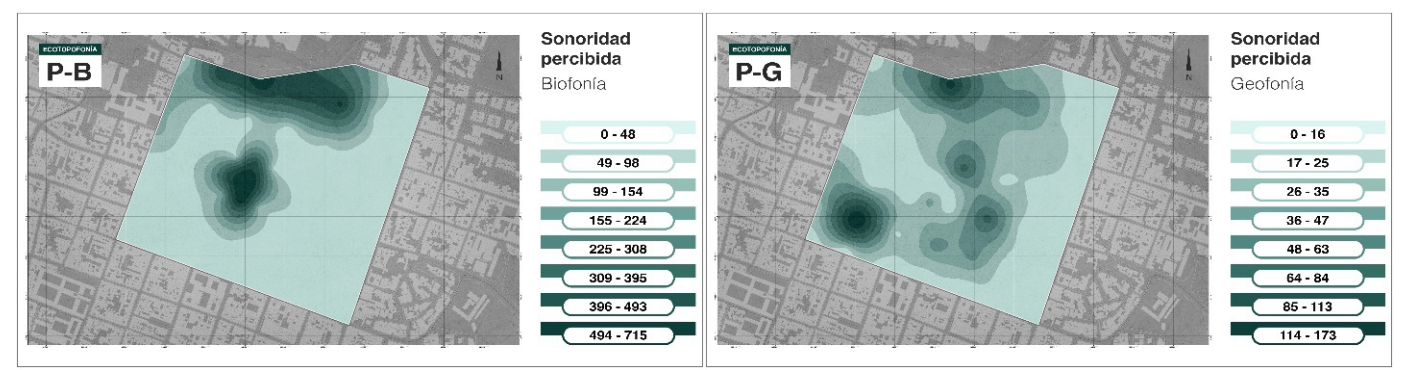

Fuente: Elaboración propia.

Vale la pena resaltar que la ecotopofonía y el sonido del transporte motorizado presentan una dinámica inversa, que se observa en ambas áreas de estudio. En ellas, el sonido del transporte motorizado opaca la biofonia producida en los parques urbanos de Popayán (Figura $N^{\circ} 13$ ), mientras que, en Venecia, el sonido del transporte motorizado es parcialmente enmascarado por la geofonía proveniente de los canales acuáticos (Figura $\mathrm{N}^{\circ} 12$ ).

En la Figura $N^{\circ} 14$ se muestran las clases principales de Venecia y Popayán durante el transcurso del día. Las secuencias temporales de antropofonía y ecotopofonía, en Venecia, alcanzan los valores máximos en el primer período (P1, 8:00-18:00) y tienden a disminuir hacia el segundo y último período de registro (P2, 18:00-00:00). El comportamiento sugiere que la producción de geofonía depende, en cierto grado, de la agitación que causan los viajes fluviales en la laguna. De otra parte, la secuencia temporal de antropofonía, en Popayán, indica valores máximos en el tercer período (P3, 12:00-14:00). Este comportamiento refleja la intensidad de desplazamientos en horas de almuerzo al mediodía. En tanto, los valores máximos en la secuencia temporal de ecotopofonía se encuentran en el primer período (P1, 8:00-10:00) y tienden a disminuir a lo largo del día, hasta el último período de registro (P5, 16:00-18:00). 
Figura $\mathrm{N}^{\circ} 14$.

Variabilidad temporal de antropofonía y ecotopofonía en las áreas de estudio.
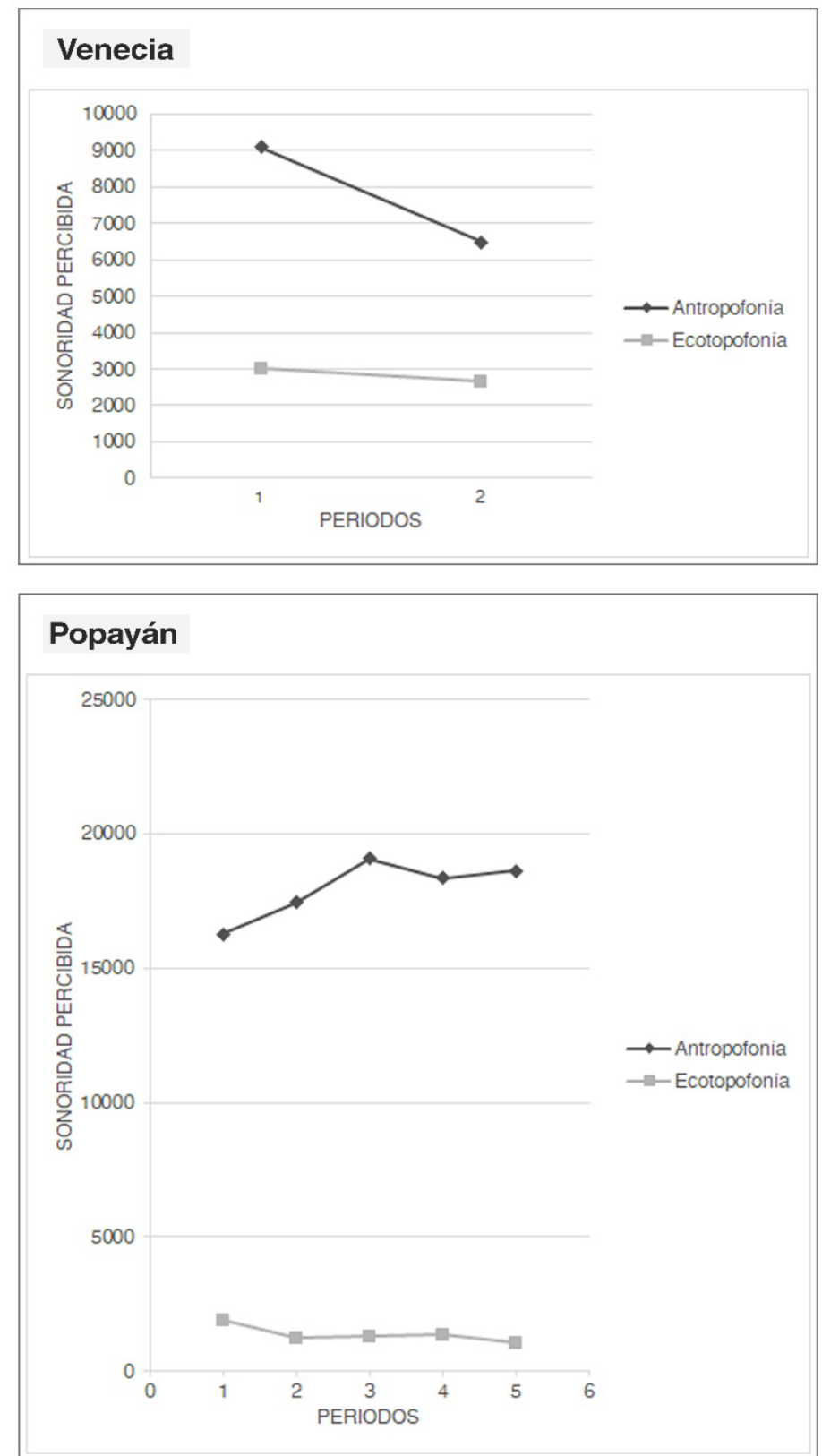

Fuente: Elaboración propia.

En la Figura $\mathrm{N}^{\circ} 15$ se representa la antropofonía y la ecotopofonía de las áreas de estudio a lo largo del día. La correlación espaciotemporal de estas clases principales de sonido es distinta para cada área de estudio: en Venecia, la geofonía dominante en los canales se percibe más a medida que la actividad humana aumenta; y en Popayán, la biofonía dominante en los parques urbanos se deja de percibir a medida que la actividad humana es mayor. 
Figura $N^{\circ} 15$.

Patrón espaciotemporal de antropofonía y ecotopofonía en las áreas de estudio



V-A Antropofonía - Sonoridad percibida

P1
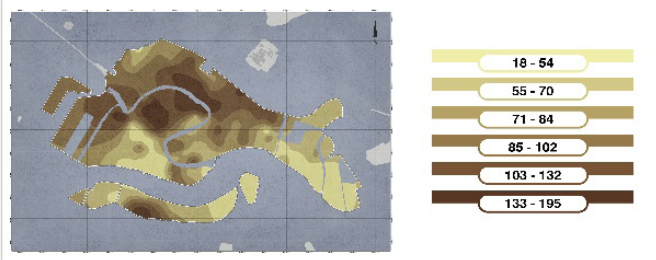

P2

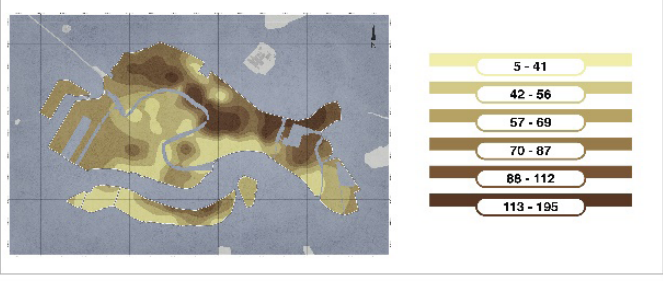

P2
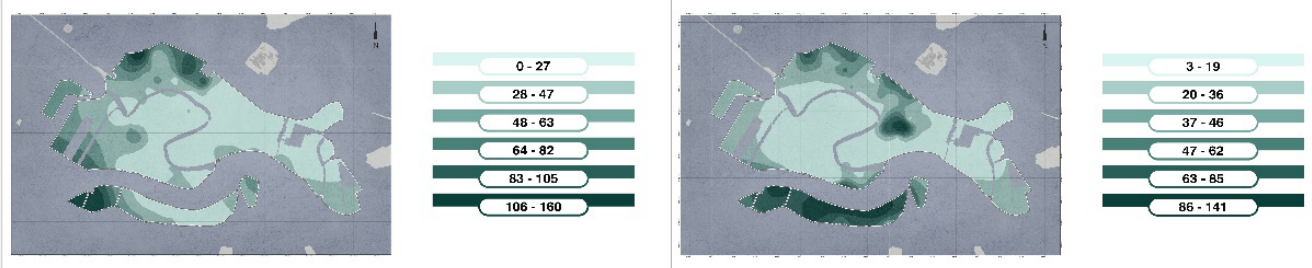

Fuente: Elaboración propia. 
El conjunto cartográfico del paisaje sonoro veneciano y payanés presentado demuestra patrones identitarios de cada ciudad estudiada, con particular coincidencia en la producción de micropaisajes sonoros que se diversifican a través de cada tipo de espacio público abierto de la ciudad (parques, plazas, vías acuáticas o terrestres, entre otros). En ese mismo sentido, conviene recordar que las Figuras $N^{\circ} 5$ y 6 evidencian que las plazas y parques públicos de Venecia y Popayán exceden el límite de $55 \mathrm{~dB}(\mathrm{~A})_{\text {Leq }}$ fijado por la WHO (1999) y la OECD (2003). Sin embargo, estos lugares son dominados por el sonido de las voces y el movimiento humano (Figuras $\mathrm{N}^{\circ} \mathrm{GV}^{\circ} \mathrm{y}$ $\mathrm{N}^{\circ} 9 \mathrm{MH}$ ), así como también por la biofonía (Figura N¹2B). Es decir, que en determinados momentos del día (Figura $\left.\mathrm{N}^{\circ} 15\right)$, el paisaje sonoro no cumple con el estándar sugerido en la normativa internacional, pero domina la ecotopofonía (producida por las aves, el viento o la lluvia; en Popayán) y la antropofonía (producida por el caminar, el hablar e incluso el cantar de las personas; en Venecia). Esto prueba que las mediciones acústicas por sí solas, no describen con exactitud el comportamiento del paisaje sonoro urbano.

\section{Discusión}

La evaluación del paisaje sonoro, en contextos urbanos diferenciados a escala global, evidenció patrones de comportamiento espaciotemporal ampliamente cambiantes. El presente estudio consta de un comparativo entre Venecia (Italia) y Popayán (Colombia), a partir del cual, se asume que la ciudad tiene la facultad de inducir los estados de transformación continua de su paisaje sonoro. En coherencia con lo planteado por Hong \& Jeon (2017), la variabilidad del paisaje sonoro de los sectores estudiados está sujeta, fundamentalmente, a las clases de sonido que resultan de las funciones predominantes del entorno urbano construido. Las cartografías elaboradas en este estudio sugieren que los sonidos de mayor dominancia están determinados por cada configuración urbana en particular (VI y MH en Venecia y TM en Popayán). En función de ello, se reconoce que el sonido del transporte motorizado a pesar de opacar fácilmente la biofonía, puede ser igualmente contrarrestado por la geofonía que producen los canales acuáticos en las áreas urbanas (Jeon et al., 2018). Por tanto, se argumenta que la calidad del paisaje sonoro depende del delicado, pero vital equilibrio, entre sus clases más representativas y experiencias sonoras derivadas.

Los paisajes sonoros dominados por el sonido del transporte motorizado coinciden con aquellas zonas del centro de Venecia y Popayán con altos SPL. Este resultado verifica que la afectación del transporte público y privado al entorno acústico de la ciudad es innegable (Raimbault \& Dubois, 2005). En tal sentido, una evaluación que trascienda la ambigua concepción del ruido urbano constituye, en verdad, un modo de mitigar la excesiva producción de paisajes sonoros subyugados al sonido de los motores.

En cualquier caso, a pesar del abrumador escenario que sugiere la acelerada degradación del paisaje sonoro urbano, es preciso considerar su antagónica capacidad para proveer de experiencias positivas a la ciudad. En conformidad con los célebres postulados de Schafer (1977), ese tipo de experiencias sonoras de gran favorabilidad se reflejan en los mapas de ecotopofonía. Sin duda, en ellos se manifiesta el enorme potencial que tiene el medio natural, al momento de repercutir adecuadamente en la impresión global del sonido urbano (Nilsson \& Berglund, 2005). 
Por otro lado, se señala que el sonido ecotopofónico confiere cualidades destacables, que, en parte, describen la identidad fónica de la ciudad (Rehan, 2016): en primer lugar, se resalta el sonido puramente biofónico, que bien podría representar a las ciudades andinas de América Latina; $y$, en segundo lugar, se pone en relieve el sonido geofónico, que podría caracterizar adecuadamente a las ciudades lagunares y/o costeras de Europa. En definitiva, estos atributos son de especial importancia en la creación de paisajes sonoros más sanos y equilibrados, que mejoren la calidad de vida de la ciudad.

Es igualmente importante anotar que la disminución de los SPL puede ser prescindible en cierta medida, ya que no necesariamente está relacionada con la calidad ambiental de la ciudad. Este estudio enfatiza en que algunos sonidos (cantos, risas, pasos y otros), a pesar de superar los límites establecidos por la WHO (1999) y la OECD (2003), pueden contribuir ampliamente a la construcción de un paisaje sonoro cautelosamente balanceado, tal y como ya habían sostenido Aletta et al. (2016). Lo anterior, contradice diversos estudios (Geraghtya \& O'Mahony, 2016; Campello et al., 2017; Vasilyev, 2017; Wang et al., 2018; Di et al., 2018), que, basados solo en parámetros físicos, niegan la interacción resultante entre el sonido de la ciudad y sus habitantes. En relación con esto, el presente trabajo pretende complementar las escalas convencionales que miden el "ruido urbano», ya que por sí solas no se adaptan a las diversas situaciones que emergen en la ciudad contemporánea.

La generación de ecotopofonías a través de la revitalización de parques y plazas públicas es una estrategia por seguir, pues permite fomentar un paisaje sonoro urbano de calidad. Esta investigación corrobora también que dicho escenario se acentúa aún más en regiones de mayor biodiversidad como Popayán (Colombia), debido a que el entorno natural predominante facilita sustancialmente la producción del sonido biofónico (Scarpelli et al., 2019). Se debe pensar, en adición, que la evaluación integral de los principales factores del paisaje sonoro prescribe directrices de utilidad para la formulación de planes que gestionen el sonido urbano de forma más acertada. En ese sentido, se argumenta que la incorporación del paisaje sonoro durante las prácticas de planeamiento urbano no es soslayable y se requiere de su implementación como un mecanismo que vele activamente por el desarrollo de una ciudad sónica más justa y equitativa para todos.

\section{Conclusiones}

El valor del paisaje sonoro radica en su capacidad para mejorar las condiciones del entorno urbano. En ese sentido, el estudio desarrollado sugiere que su intervención debe trascender la clásica evaluación de los niveles de ruido (SPL), que, en el contexto latinoamericano y/o europeo, no siempre cumplen con los estándares fijados a escala internacional. De hecho, es a partir de la amplia comprensión de los patrones de comportamiento del paisaje sonoro, en momentos y lugares específicos, que en realidad se puede generar acciones territoriales sólidas para dignificar la vida urbana.

El equilibrio del entorno acústico está directamente relacionado con el tratamiento del espacio público abierto, sobre todo parques y plazas públicas. Estos ámbitos, a fin de cuentas, deben promover la producción paisajes sonoros apropiados para la ciudadanía. En tal sentido, aquí se 
defiende la provisión de paisajes sonoros con un balance saludable entre ecotopofonía y antropofonía, de modo que sea una función adicional que se tome en consideración durante los procesos de planeamiento urbano. En efecto, lo anterior puede ser posible, siempre que se fomenten experiencias sonoras congruentes con la singularidad de cada contexto urbano, para que, en el largo alcance, logren aportar al bienestar social de la población.

En adición, este estudio presenta dos potenciales limitantes: la primera, es la escala geográfica, pues el área de estudio de Venecia supera en 10 veces el tamaño del área de estudio de Popayán; y la segunda, es el método de recolección de datos, si bien se tienen al menos 100 puntos de muestreo para cada área de estudio, los registros no son del todo estandarizados, ya que estos oscilan en muestreos de entre 3 a 15 minutos y en períodos de 6 a 2 horas según cada caso. El presente trabajo garantiza el análisis comparativo realizado, pero los resultados no pueden extrapolarse a una relación directa entre áreas de igual extensión geográfica y volumen de datos examinados. En consecuencia, solo es posible comentar diferencias y similitudes parciales entre los casos estudiados, y conforme a los resultados previamente expuestos.

Finalmente, se recomienda tener en cuenta las limitaciones mencionadas en futuras comparaciones sobre paisajes sonoros urbanos, de modo que se involucren más ciudades y se amplié la escala de evaluación espaciotemporal empleada. Asimismo, es aconsejable profundizar en la comprensión del paisaje sonoro urbano a través de entrevistas que detallen los significados connotativos y matices semánticos del sonido de la ciudad, pues al paisaje es válido aproximarse tanto por la vía cuantitativa como por la cualitativa (Nogué et al., 2010).

\section{Agradecimientos}

El desarrollo de este material fue respaldado por el Centro de Estudios Urbanos de la Institución Universitaria Colegio Mayor del Cauca (IUCMC). Por ello, se agradece a todo su equipo de trabajo y, en particular, a sus directores, Germán Chamorro y Andrés Córdoba, por su desinteresado apoyo a esta investigación. También se extiende un sentido de gratitud a las directivas máximas de la IUCMC, su rector, Héctor Sánchez Collazos, y su vicerrectora académica, Paola Umaña Aedo, por confiar siempre en este trabajo. Del mismo modo, se expresan sinceros agradecimientos a la artista conceptual, Ivana Blanco Gross, cuya trayectoria artística ayudó a consolidar la realización de este estudio y al Dr. Prof. Valerià Paül, por siempre motivar a construir un proceso académico altamente responsable. Por último, se expresa especial gratitud a los dos evaluadores anónimos, dado que sus contribuciones fueron sustanciales en la mejora de este artículo, e igualmente a todo el equipo editorial de la Revista de Geografía Norte Grande, por la oportunidad concedida.

\section{Referencias}

AGNEW, J. Space and place. In AGNEW, J; LIVINGSTONE, D. (eds.), Handbook of Geographical Knowledge. London: Sage, 2011, p. 316-331.

ALCALDÍA DEL MUNICIPIO DE POPAYÁN. Plan Especial de Manejo y Protección del Sector Antiguo de Popayán. Popayán: Diario Oficial de la República de Colombia, 2010. 
ALETTA, F., KANG, J. \& AXELSSON, Ö. Soundscape Descriptors and a Conceptual Framework for Developing Predictive Soundscape Models. Landscape and Urban Planning, 2016, Vol. 149, p. 6574. https://doi.org/10.1016/j.landurbplan.2016.02.001

ALETTA, F., OBERMAN, T., MITCHELL, A., ERFANIAN, M., LIONELLO, M. \& KANG, J. Associations Between Soundscape Experience and Self-Reported Wellbeing in Open Public Urban Spaces: A Field Study. The Lancet, 2019, Vol. 394, p. S17. https://doi.org/10.1016/S0140-6736(19)32814-4

BROWN, A.L., KANG, J. \& GJESTLAND, T. Towards Standardization in Soundscape Preference Assessment. Applied Acoustics, 2011, Vol. 72, N6, p. 387-392. https://doi.org/10.1016/j. apacoust.2011.01.001

BRUNDTLAND, G.H., KHALID, M., AGNELLI, S. \& CHIDZERO, B. Our Common Future. Oxford: Oxford University Press, 1987.

CAIN, R., JENNINGS, P. \& POXON, J. The Development and Application of the Emotional Dimensions of a Soundscape. Applied acoustics, 2013, Vol. 74, N², p. 232-239. https://doi.org/10.1016/j. apacoust.2011.11.006

CAMPELLO-VICENTE, H., PERAL-ORTS, R., CAMPILLO-DAVO, N. \& VELASCO-SANCHEZ, E. The Effect of Electric Vehicles on Urban Noise Maps. Applied Acoustics, 2017, Vol. 116, p. 59-64. https:// doi.org/10.1016/j.apacoust.2016.09.018

CERWÉN, G. Urban Soundscapes: A Quasi-Experiment in Landscape Architecture. Landscape Research, 2016, Vol. 41, N5, p. 481-494. https://doi.org/10.1080/01426397.2015.1117062

CERWÉN, G., KREUTZFELDT, J. \& WINGREN, C. Soundscape Actions: A Tool for Noise Treatment Based on Three Workshops in Landscape Architecture. Frontiers of Architectural Research, 2017, Vol. 6, N4, p. 504-518. https://doi.org/10.1016/j.apacoust.2016.10.002

COMUNE DI VENEZIA. Piano di Assetto del Territorio del Comune di Venezia. Venezia: Bollettino Ufficiale della Regione Autónoma Friuli-Venezia Giulia, 2014.

COMUNE DI VENEZIA. Popolazione Residente dal 2000 al 2019 [in linea]. Italia: Comune di Venezia, 2019. [Data di consultazione: 27 settembre 2020]. Disponibile presso: https://www.comune.venezia.it/it/content/serie-storiche

CÓRDOBA, A. GALVIS O. \& ORDÓÑEZ, H. Camuflados: asesinados por el Ejército Nacional. Popayán: Editorial de la Universidad del Cauca, 2016.

DE LA PRIDA, D., PEDRERO, A., NAVACERRADA, M.Á \& DÍAZ, C. Relationship between the Geometric Profile of the City and the Subjective Perception of Urban Soundscapes. Applied Acoustics, 2019, Vol. 149, p. 74-84. https://doi.org/10.1016/j.apacoust.2019.01.025

DEPARAMENTO NACIONAL ADMINISTRATIVO DE ESTAdístICA (DANE). Censo Nacional de Población y Vivienda [en línea]. Colombia: DANE, 2018. [Fecha de consulta: 23 de marzo de 2020]. Dis- 
ponible en: https://www.dane.gov.co/index.php/estadisticas-por-tema/demografia-y-poblacion/ proyecciones-de-poblacion

DI, H., LIU, X., ZHANG, J., TONG, Z., JI, M., LI, F., FENG, T. \& MA Q. Estimation of the Quality of an Urban Acoustic Environment Based on Traffic Noise Evaluation Models. Applied Acoustics, 2018, Vol. 141, p. 115-124. https://doi.org/10.1016/j.apacoust.2018.07.010

FARINA, A. \& PIERETTI, N. Acoustic Codes in Action in a Soundscape Context. Biosemiotics, 2014, Vol. 7, N², p. 321-328. https://doi.org/10.1007/s12304-014-9213-0

FILIPAN, K., DE COENSEL, B., AUMOND, P., CAN, A., LAVANDIER, C. \& BOTTELDOOREN, D. Auditory Sensory Saliency as a Better Predictor of Change than Sound Amplitude in Pleasantness Assessment of Reproduced Urban Soundscapes. Building and Environment, 2019, Vol. 148, p. 730-741. https://doi.org/10.1016/j.buildenv.2018.10.054

GERAGHTY, D. \& O'MAHONY, M. Investigating the Temporal Variability of Noise in an Urban Environment. International Journal of Sustainable Built Environment, 2016, Vol. 5, №1, p. 34-45. https:// doi.org/10.1016/j.ijsbe.2016.01.002

GOOVAERTS, P. Geostatistics in Soil Science: State-Of-The-Art and Perspectives. Geoderma, 1999, Vol. 89, No1-2, p. 1-45. https://doi.org/10.1016/S0016-7061(98)00078-0

GOZALO, G.R., CARMONA, J.T., BARRIGÓN, J.M., VÍLCHEZ-GÓMEZ, R. \& GÓMEZ, V. Relationship Between Objective Acoustic Indices and Subjective Assessments for The Quality of Soundscapes. Applied Acoustics, 2015, Vol. 97, p. 1-10. https://doi.org/10.1016/j.apacoust.2015.03.020

GRIJALBA, J. \& CÓRDOBA, A. Ciudad sónica: el caso paradigmático de Popayán (Colombia). Popayán: Editorial de la Universidad del Cauca, 2020.

HARMAN, B.I., KOSEOGLU, H. \& YIGIT, C.O. Performance Evaluation of IDW, Kriging and Multiquadric Interpolation Methods in Producing Noise Mapping: A Case Study at the City of Isparta, Turkey. Applied Acoustics, 2016, Vol. 112, p. 147-157. https://doi.org/10.1016/j.apacoust.2016.05.024

HERMIDA, L. \& PAVÓN, I. Spatial Aspects in Urban Soundscapes: Binaural Parameters Application in the Study of Soundscapes from Bogotá-Colombia and Brasília-Brazil. Applied Acoustics, 2019, Vol. 145, p. 420-430. https://doi.org/10.1016/j.apacoust.2018.10.011

HONG, J.Y. \& JEON, J.Y. Exploring Spatial Relationships among Soundscape Variables in Urban Areas: A Spatial Statistical Modelling Approach. Landscape and Urban Planning, 2017, Vol. 157, p. 352-364. https://doi.org/10.1016/j.landurbplan.2016.08.006

ISO (INTERNATIONAL ORGANIZATION FOR STANDARDIZATION). ISO 12913-1: 2014. AcousticsSoundscape-Part 1: Definition and Conceptual Framework. Switzerland: ISO, 2014. 
JEON, J.Y. \& JO, H.I. Effects of Audio-Visual Interactions on Soundscape and Landscape Perception and their Influence on Satisfaction with The Urban Environment. Building and Environment, 2020, Vol. 169, N¹06544, p. 1-42. https://doi.org/10.1016/j.buildenv.2019.106544

JEON, J.Y., HONG, J.Y., LAVANDIER, C., LAFON, J., AXELSSON, Ö. \& HURTIG, M. A Cross-National Comparison in Assessment of Urban Park Soundscapes in France, Korea, and Sweden Through Laboratory Experiments. Applied Acoustics, 2018, Vol. 133, p. 107-117. https://doi.org/10.1016/j. apacoust.2017.12.016

JIA, Y., MA, H. \& KANG, J. Characteristics and Evaluation of Urban Soundscapes Worthy of Preservation. Journal of Environmental Management, 2020, Vol. 253, N¹09722, p 1-10. https://doi. org/10.1016/j.jenvman.2019.109722

KANG, J. \& SCHULTE-FORTKAMP B. Soundscape and the Built Environment. Boca Ratón: CRC Press, 2016.

KOGAN, P., ARENAS, J.P., BERMEJO, F., HINALAF, M. \& TURRA, B. A Green Soundscape Index (GSI): The Potential of Assessing the Perceived Balance Between Natural Sound and Traffic Noise. Science of the Total Environment, 2018, Vol. 642, p. 463-472. https://doi.org/10.1016/j.scitotenv.2018.06.023

KOGAN, P., TURRA, B., ARENAS, J.P \& HINALAF, M. A Comprehensive Methodology for The Multidimensional and Synchronic Data Collecting in Soundscape. Science of The Total Environment, 2017, Vol. 580, p. 1068-1077. https://doi.org/10.1016/j.scitotenv.2016.12.061

LEUS, M. \& HERSSENS, J. The Soundscapes of Antwerp: A Study on the Acoustic Genius Loci. Energy Procedia, 2015, Vol. 78, p. 25-30. https://doi.org/10.1016/j.egypro.2015.11.109

LI, C., LIU, Y. \& HAKLAY, M. Participatory Soundscape Sensing. Landscape and Urban Planning, 2018, Vol. 173, p. 64-69. https://doi.org/10.1016/j.landurbplan.2018.02.002

LIU, F. \& KANG, J. A Grounded Theory Approach to The Subjective Understanding of Urban Soundscape in Sheffield. Cities, 2016, Vol. 50, p. 28-39. https://doi.org/10.1016/j.cities.2015.08.002

LIU, J., KANG, J., LUO, T. \& BEHM, H. Landscape Effects on Soundscape Experience in City Parks. Science of the Total Environment, 2013a, Vol. 454, p. 474-481. https://doi.org/10.1016/j.scitotenv.2013.03.038

LIU, J., KANG, J., LUO, T., BEHM, H. \& COPPACK, T. Spatiotemporal Variability of Soundscapes in a Multiple Functional Urban Area. Landscape and Urban Planning, 2013b, Vol. 115, p. 1-9. https://doi. org/10.1016/j.landurbplan.2013.03.008

LIU, J., WANG, Y., ZIMMER, C., KANG, J. \& YU, T. Factors Associated with Soundscape Experiences in Urban Green Spaces: A Case Study in Rostock, Germany. Urban Forestry \& Urban Greening, 2019, Vol. 37, p. 135-146. https://doi.org/10.1016/j.ufug.2017.11.003 
MARISTANY, A., LÓPEZ, M.R. \& RIVERA, C.A. Soundscape Quality Analysis by Fuzzy Logic: A Field Study in Cordoba, Argentina. Applied Acoustics, 2016, Vol. 111, p. 106-115. https://doi.org/10.1016/j. apacoust.2016.04.013

MAZORRA, M.I., CÓRDOBA, A.A, \& ZÚÑIGA, F.L. Expansión urbana y ordenación territorial: la urbanización fuera de plan en Popayán, Colombia. En CARRIÓN, A.; LÓPEZ SANDOVAL, M.F. (eds), Ciudades intermedias y nueva ruralidad. Quito: FLACSO Ecuador, 2021, p. 209-232.

MODAK, S. Venice Looks to Limit Tourist Numbers with New Measures [online]. Condé Nast Traveler, 2017. [Accessed 27 September 2020]. https://www.cntraveler.com/story/venice-looks-to-limittourist-numbers-with-new-measures

MORALES, S. Sociedades en Movimientos. Popayán: Territorios ocultos en la historia de la ciudad, 2020.

NILSSON, M. \& BERGLUND, B. Assessment of Outdoor Soundscapes in Quiet Areas. The Journal of the Acoustical Society of America, 2005, Vol. 117, N4, p. 2592-2592. https://doi.org/10.1121/1.4777308

NOGUÉ, J., PUIGBERT, L., SALA, P. \& BRETCHA, G. Paisatge i participació ciutadana. Catalunya: Observatori del Paisatge de Catalunya/Direcció General de Participació Ciutadana del Departament d'Interior, Relacions Institucionals i Participació Ciutadana de la Generalitat de Catalunya, 2010.

OLDONI, D., DE COENSEL, B., BOES, M., RADEMAKER, M., DE BAETS, B., VAN RENTERGHEM T. \& BOTTELDOOREN, D. A Computational Model of Auditory Attention for Use in Soundscape Research. The Journal of the Acoustical Society of America, 2013, Vol. 134, N¹, p. 852-861. https:// doi.org/10.1121/1.4807798

ORGANISATION FOR ECONOMIC CO-OPERATION AND DEVELOPMENT (OECD). Environmental Indicators, Development, Measurement and Use. París: OECD, 2003.

PIJANOWSKI, B., FARINA, A., GAGE, S., DUMYAHN, S. \& KRAUSE, B. What is Soundscape Ecology? An Introduction and Overview of An Emerging New Science. Landscape Ecology, 2011, Vol. 26, N9, p. 1213-1232. https://doi.org/10.1007/s10980-011-9600-8

RAIMBAULT, M \& DUBOIS, D. Urban Soundscapes: Experiences and Knowledge. Cities, 2005, Vol. 22, N5, p. 339-350. https://doi.org/10.1016/j.cities.2005.05.003

REHAN, R.M. The Phonic Identity of The City Urban Soundscape for Sustainable Spaces. HBRC Journal, 2016, Vol. 12, N³, p. 337-349. https://doi.org/10.1016/j.hbrcj.2014.12.005

ROMERO, V.P., MAFFEI, L., BRAMBILLA, G. \& CIABURRO, G. Modelling the Soundscape Quality of Urban Waterfronts by Artificial Neural Networks. Applied Acoustics, 2016, Vol. 111, p. 121-128. https://doi.org/10.1016/j.apacoust.2016.04.019 
SCARPELLI, M., RIBEIRO M.C., TEIXEIRA, F.Z., YOUNG R.J. \& TEIXEIRA, C.P. Gaps in Terrestrial Soundscape Research: It's Time to Focus on Tropical Wildlife. Science of the Total Environment, 2019, Vol. 707, p. 1-22. https://doi.org/10.1016/j.scitotenv.2019.135403

SCHAFER, R. M. The soundscape: Our sonic environment and the tuning of the world. New York: Knopf, 1977.

SOUTHWORTH, M. The sonic environment of cities. Thesis, Massachusetts Institute of Technology, Cambridge: United States, 1969.

STANNERS, D. \& BOURDEAU, P. Europe's environment: the Dobřiš assessment [online]. Luxembourg: Office for Official Publications of the European Communities, 1995. Available in: https:// www.eea.europa.eu/publications/92-826-5409-5

SUN, K., DE COENSEL, B., FILIPAN, K., ALETTA, F., VAN RENTERGHEM, T., DE PESSEMIER T., JOSEPH, W. \& BOTTELDOOREN, D. Classification of Soundscapes of Urban Public Open Spaces. Landscape and Urban Planning, 2019, Vol. 189, p. 139-155. https://doi.org/10.1016/j.landurbplan.2019.04.016

SZEREMETA, B. \& ZANNIN, P. Analysis and Evaluation of Soundscapes in Public Parks Through Interviews and Measurement of Noise. Science of the Total Environment, 2009, Vol. 407, №24, p. 6143-6149. https://doi.org/10.1016/j.scitotenv.2009.08.039

TORIJA MARTINEZ, A., LI, Z. \& SELF, R. Effects of A Hovering Unmanned Aerial Vehicle on Urban Soundscapes Perception. Transportation Research Part D: Transport and Environment, 2020, Vol. 78, No102195, p. 1-20. https://doi.org/10.1016/j.trd.2019.11.024

TORIJA, A.J., RUIZ, D.P. \& RAMOS-RIDAO, Á. Required Stabilization Time, Short-Term Variability, and Impulsiveness of The Sound Pressure Level to Characterize the Temporal Composition of Urban Soundscapes. Applied Acoustics, 2011, Vol. 72, N²-3, p. 89-99. https://doi.org/10.1016/j. apacoust.2010.09.011

TRUAX, B. Acoustic space, architecture, and acoustic ecology. In: Proceedings of Architecture, Music, Acoustics. International Cross-Disciplinary Conference. Toronto: Ryerson University, 2006, p. 8-10.

United Nations Educational, Scientific and Cultural Organization (UNESCO). Text of the Convention for the Safeguarding of the Intangible Cultural Heritage [online]. París: UNESCO, 2009. [ACcessed 27 September 2020]. Available in: https://ich.unesco.org/doc/src/2003_Convention_Basic_Texts-_2020_version-EN.pdf

United Nations Educational, Scientific and Cultural Organization (UNESCO). Popayan UNESCO City of Gastronomy [online]. París: UNESCO, 2009. [Accessed 27 September 2020]. https://unesdoc.unesco.org/ark:/48223/pf0000159284/PDF/159284eng.pdf.multi 
VAN RENTERGHEM, T., VANHECKE, K., FILIPAN, K., SUN, K., DE PESSEMIER, T., DE COENSEL, B., JOSEPH, W. \& BOTTELDOOREN, D. Interactive Soundscape Augmentation by Natural Sounds in A Noise Polluted Urban Park. Landscape and Urban Planning, 2020, Vol. 194, p. 103705. https://doi. org/10.1016/j.landurbplan.2019.103705

VASILYEV, A.V. New Methods and Approaches to Acoustic Monitoring and Noise Mapping of Urban Territories and Experience of It Approbation in Conditions of Samara Region of Russia. Procedia Engineering, 2017, Vol. 176, p. 669-674. https://doi.org/10.1016/j.proeng.2017.02.311

VOGIATZIS, K. \& REMY, N. From Environmental Noise Abatement to Soundscape Creation Through Strategic Noise Mapping in Medium Urban Agglomerations in South Europe. Science of the total Environment, 2014, Vol. 482, p. 420-431. https://doi.org/10.1016/j.scitotenv.2013.07.098

WANG, H., CHEN, H. \& CAI, M. Evaluation of an urban traffic Noise-Exposed population based on points of interest and noise maps: The case of Guangzhou. Environmental pollution, 2018, Vol. 239, p. 741-750. https://doi.org/10.1016/j.envpol.2017.11.036

WATTS, G.R. \& PHEASANT, R.J. Tranquillity in the Scottish Highlands and Dartmoor National ParkThe importance of soundscapes and emotional factors. Applied Acoustics, 2015, Vol. 89, p. 297305. https://doi.org/10.1016/j.apacoust.2014.10.006

WEI, W., VAN RENTERGHEM, T., DE COENSEL, B. \& BOTTELDOOREN, D. Dynamic Noise Mapping: A Map-Based Interpolation between Noise Measurements with High Temporal Resolution. Applied Acoustics, 2016, Vol. 101, p. 127-140. https://doi.org/10.1016/j.apacoust.2015.08.005

WORLD HEALTH ORGANIZATION (WHO). Environmental Noise Guidelines for the European Region. Copenhagen. Copenhague: WHO, 2018. 
